\title{
An improved algorithm for the shallow water equations model reduction: Dynamic Mode Decomposition vs POD
}

\author{
D. A. Bistrian ${ }^{1, * \dagger}$ and I. M. Navon ${ }^{2}$ \\ ${ }^{1}$ Department of Electrical Engineering and Industrial Informatics, University “Politehnica” of Timisoara, 331128 \\ Hunedoara, Romania \\ ${ }^{2}$ Department of Scientific Computing, Florida State University, Tallahassee, FL, 32306-4120, USA
}

\begin{abstract}
SUMMARY
We propose an improved framework for dynamic mode decomposition (DMD) of 2-D flows for problems originating from meteorology when a large time step acts like a filter in obtaining the significant Koopman modes, therefore, the classic DMD method is not effective. This study is motivated by the need to further clarify the connection between Koopman modes and proper orthogonal decomposition (POD) dynamic modes. We apply DMD and POD to derive reduced order models (ROM) of the shallow water equations. Key innovations for the DMD-based ROM introduced in this paper are the use of the Moore-Penrose pseudoinverse in the DMD computation that produced an accurate result and a novel selection method for the DMD modes and associated amplitudes and Ritz values. A quantitative comparison of the spatial modes computed from the two decompositions is performed, and a rigorous error analysis for the ROM models obtained is presented. Copyright (C) 2015 John Wiley \& Sons, Ltd.
\end{abstract}

Received 31 July 2014; Revised 19 December 2014; Accepted 1 March 2015

KEY WORDS: dynamic mode decomposition; proper orthogonal decomposition; model order reduction; shallow water equations

\section{INTRODUCTION}

The modal decomposition of fluid dynamics is a frequently employed technique, capable of providing tools for studying dominant and coherent structures in turbulent flows. The coherent structures [1-3] represent spatially or temporally evolving vortical motions, either growing with one rate, oscillating with one frequency or containing the largest possible kinetic energy. A complex turbulent flow often consists of a superposition of such coherent structures, whose development is responsible for the bulk mass, energy transfer or hydrodynamic instability.

Among several snapshot-based model order reduction (MOR) modal decomposition methods, proper orthogonal decomposition (POD) and dynamic mode decomposition (DMD) have been widely applied to study the physics of the dynamics of the flows in different applications. The MOR using the method of POD has been illustrated on a variety of examples ranging from fluid mechanics (Luchtenburg and Rowley [4], Liberge and Hamdouni [5]), turbulent flows and oceanography (Wang et al. [6], Abramov and Majda [7], Osth et al. [8]) or engineering structures (Mariani and Dessi [9] and Buljak and Maier [10]). More recently, the POD approach has been incorporated, for reduced order modelling purposes, within an unstructured mesh finite-element ocean model by Du et al. [11], Fang et al. [12] and Stefanescu and Navon [13]. POD proved to be an effective technique also in inverse problems, as demonstrated in the work of Winton et al. [14], Chen et al. $[15,16]$ and Cao et al. $[17,18]$.

*Correspondence to: D. A. Bistrian, Revolutiei Str. Nr.5, 331128 Hunedoara, Romania.

${ }^{\dagger}$ E-mail: diana.bistrian@upt.ro 
Several types of global modes are considered. Linear global eigenmodes are small-amplitude perturbations that grow or decay exponentially and pulsate with one frequency. They are used in hydrodynamic stability analysis [19]. Balanced modes are used to construct low-dimensional models of large-scale flow systems in order to capture the relation between input disturbances and the output sensors used for flow measurements. Projecting the original linear system onto modes results in a high-fidelity model that accurately reproduces the input-output dynamics of the model [20,21].

Koopman modes represent spatial flow structures with time-periodic motion, which are optimal in resolving oscillatory behavior. They have been increasingly used because they provide a powerful way of analyzing nonlinear flow dynamics using linear techniques (see e.g. the work of Bagheri [22], Mezic [23], Rowley et al. [24]). The DMD generalizes the global stability modes and approximates the eigenvalues of the Koopman operator [25]. The Koopman modes are extracted from the data snapshots, and a unique frequency is associated with each mode. This is of major interest for fluid dynamics applications where phenomena occurring at different frequencies must be individualized.

The application of POD is primarily limited to flows whose coherent structures can be hierarchically ranked in terms of their energy content. But there are situations when the energy content is not a sufficient criterion to accurately describe the dynamical behaviour of the aforementioned flows. Instead, DMD links the dominant flow features by a representation in the amplitudes-temporal dominant frequencies space.

A comparative analysis of POD and DMD has been performed in the literature to identify which of these decomposition techniques is more efficient. Recent studies performed in various fields have demonstrated that these are complementary methods contributing to the identification of systems in different ways. Simultaneous application of the two methods provides an a priori knowledge of the dynamics of the system. For example, Semeraro et al. [26] present a comparative analysis of POD-DMD computed from experimental data of a turbulent jet. The extracted DMD modes exhibit many similarities with the POD modes, and the flapping mode was easily identified using both methods. The transition to unsteadiness and the dynamics of weakly turbulent natural convection in a differentially heated 3-D cavity was successfully investigated by Soucasse et al. [27] by modal decomposition. In a novel approach manner, Frederich and Luchtenburg [28] consider the POD and DMD. They show how the correlation matrix, which is needed for POD, can be re-used in the computation of the DMD modes. In the field of aerodynamics, Muld et al. [29] applied POD and DMD to extract the most dominant flow structures of a simulated flow in the wake of a high-speed train model. They perform a comparison between the modes from the two different decomposition methods.

The present study is motivated by the need to further clarify the connection between Koopman modes and POD dynamic modes, as well as address their physical significance, in modal decomposition of flows with large time span. In general, for problems occurring in meteorology [30, 31] or oceanography $[32,33]$, the use of large time step for observables is justified. It was realized that application of DMD to large time steps observations or to small time steps observations when the increments are smaller then the experimental noise is subject to several predicaments; therefore, the classic DMD method is not effective.

In this work, a new approach is proposed to derive an improved DMD-based procedure that is able to extract dynamically relevant flow features from time-resolved experimental or numerical data. Our objective is to employ the improved DMD technique in parallel with the classic DMD method and POD, in order to analyze which of these procedures better highlight the coherent structures of the flow dynamics. The novelty introduced in this paper resides in application of the improved DMD technique to problems originating from meteorology, when numerical or experimental data snapshots are captured with large time steps. The modes selection, which is central in model reduction, represents the subject that we aim to investigate in this paper. We propose a new criterion of selecting the optimal Koopman modes. Additionally, we present a rigorous error analysis for the ROM models obtained by POD and the improved DMD and we also compare the relative computational efficiency of the aforementioned ROM methods.

The remainder of this article is organized as follows. The procedure of numerical data acquisition is presented in Section 2. In Section 3, we recall the principles governing the DMD and we give the description of the improved DMD algorithm. In particular, we discuss the implementation of 
the proposed method for 2-D flows and the criterion for optimal selection of the Koopman modes. The principles governing the POD are discussed in detail in Section 4, which includes also the algorithm for computing the 2-D proper orthogonal modes. These strategies are applied to the shallow water equation (SWE) model in Section 5, along with a qualitative analysis of Koopman and POD modes, while the reduced order models (ROM) obtained by involving the DMD and POD expansion of the variables are discussed in detail in Section 6. Summary and conclusions are drawn in the final section.

\section{NUMERICAL DATA ACQUISITION}

We consider a bounded open domain $\Omega \subset \mathbb{R}^{3}$ and let $L^{2}(\Omega)$ be the Hilbert space of square integrable vector functions over $\Omega$, associated with the energy norm $\|w\|_{L^{2}}=(w, w)_{L^{2}}^{1 / 2}$ and the standard inner product $(v, w)_{L^{2}}=\int_{\Omega} v \cdot w d z$. Let $H_{\nabla}$ be the Hilbert space of divergence free functions given by

$$
H_{\nabla}=\left\{w \in L^{2}(\Omega) \mid \nabla \cdot w=0 \text { in } \Omega, w \cdot \vec{n}=0 \text { on } \partial \Omega\right\},
$$

where $\vec{n}$ is the outward normal to the boundary. We define $H^{d}(\Omega) \subset L^{2}(\Omega)$ to be the Hilbert space of functions $w$ with $d$ distributional derivatives $\nabla w_{i}, 1 \leqslant i \leqslant d$, which are all square integrable. Let $\mathbb{V}$ be the Hilbert space

$$
\mathbb{V}=\left\{w \in H_{\nabla} \mid w \in H^{1}(\Omega), w=0, \frac{\partial w}{\partial \vec{n}}=0, \text { on } \partial \Omega\right\},
$$

with norm $\|w\|_{\mathbb{V}}=(w, w)_{\mathbb{V}}^{1 / 2}$ and the inner product $(v, w)_{\mathbb{V}}=\sum_{i=1}^{d}\left(\nabla v_{i}, \nabla w_{i}\right)$.

In the Cartesian coordinates formulation, we suppose there exists a time-dependent flow $w=$ $(u, v, h)(x, y, t) \in \mathbb{V}$ and a given initial flow $w(x, y, 0)=\left(u_{0}, v_{0}, h_{0}\right)(x, y)$, which are solutions of the Saint Venant equations, also called the SWE [34],

$$
\begin{gathered}
u_{t}+u u_{x}+v u_{y}+\eta_{x}-f v=0, \\
v_{t}+u v_{x}+v v_{y}+\eta_{y}+f u=0, \\
\eta_{t}+(\eta u)_{x}+(\eta v)_{y}=0,
\end{gathered}
$$

where $u(x, y, t)$ and $v(x, y, t)$ are the velocity components in the $x$ and $y$ axis, respectively, $\eta(x, y, t)=g h(x, y, t)$ is the geopotential height, $h(x, y, t)$ represents the depth of the fluid, $f$ is the Coriolis factor and $g$ is the acceleration of gravity. Subscripts represent the derivatives with respect to time and the streamwise and spanwise coordinates.

We consider that the reference computational configuration is the rectangular 2-D domain $\Omega=$ $\left[0, L_{\max }\right] \times\left[0, D_{\max }\right]$. The model (3)-(5) is considered here in a $\beta$-plane assumption [35], in which the effect of the earth's sphericity is modelled by a linear variation in the Coriolis factor

$$
f=\widehat{f}+\frac{\beta}{2}\left(2 y-D_{\max }\right),
$$

where $\widehat{f}$ and $\beta$ are constants and $L_{\max }$ and $D_{\max }$ are the dimensions of the rectangular domain of integration $\Omega$.

The SWE have been used for a wide variety of hydrological and geophysical fluid dynamics phenomena such as tide-currents [36], pollutant dispersion [37], storm-surges or tsunami wave propagation [38].

The test problem used in this paper consisted of the nonlinear SWE (3)-(5) in a channel on the rotating earth, associated with periodic boundary conditions in the $x$-direction and solid wall boundary condition in the $y$-direction: 


$$
w(0, y, t)=w\left(L_{\max }, y, t\right), v(x, 0, t)=v\left(x, D_{\max }, t\right)=0 .
$$

The initial condition I1 introduced by Grammeltvedt [39] was adopted as the initial height field, has been tested by different researchers (Cullen and Morton [40], Navon [41], Stefanescu and Navon [13] and Fang et al. [42]), that is,

$$
h_{0}(x, y)=H_{0}+H_{1} \tanh \left(\frac{9\left(D_{\max } / 2-y\right)}{2 D_{\max }}\right)+H_{2} \sin \left(\frac{2 \pi x}{L_{\max }}\right) \cosh ^{-2}\left(\frac{9\left(D_{\max } / 2-y\right)}{D_{\max }}\right),
$$

which propagates the energy in wave number one, in the streamwise direction. Using the geostrophic relationship, $u=-h_{y}(g / f), v=h_{x}(g / f)$, the initial velocity fields are derived as:

$$
\begin{aligned}
u_{0}(x, y)= & -\frac{g}{f} \frac{9 H_{1}}{2 D_{\max }}\left(\tanh ^{2}\left(\frac{9 D_{\max } / 2-9 y}{2 D_{\max }}\right)-1\right) \\
& -\frac{18 g}{f} H_{2} \sinh \left(\frac{9 D_{\max } / 2-9 y}{D_{\max }}\right) \frac{\sin \left(\frac{2 \pi x}{L_{\max }}\right)}{D_{\max } \cosh ^{3}\left(\frac{9 D_{\max } / 2-9 y}{D_{\max }}\right)}, \\
v_{0}(x, y)= & 2 \pi H_{2} \frac{g}{f L_{\max }} \cos \left(\frac{2 \pi x}{L_{\max }}\right) \cosh ^{-2}\left(\frac{9\left(D_{\max } / 2-y\right)}{D_{\max }}\right) .
\end{aligned}
$$

In developing a higher-order scheme for approximating the quadratically nonlinear terms that appear in the equations of hydrological dynamics, we have followed the approach used by Navon [35], which implements a two-stage finite-element Numerov-Galerkin method for integrating the nonlinear SWE on a $\beta$-plane limited-area domain. In the aforementioned paper, a two-stage Galerkin method combined with a high-accuracy compact (Numerov) approximation to the first derivative is presented. This method when applied to meteorological and oceanographic problems not only gives an accurate phase propagation but also handles nonlinearities well. The accuracy of temporal and spatial discretization scheme equals or exceeds $\mathcal{O}\left(k^{2}, h^{4-8}\right)$. The use of numerical integration methods to study the behaviour of theoretical models in oceanography or to predict the evolution of an actual state is subject to several predicaments, one of the major difficulties being nonlinear computational instability of the finite difference analogues of the governing partial differential equations. The approach adopted in the work noted earlier involves the use of a weighted selective lumping scheme in the finite-element method, combined with a successive overrelaxation iterative method for solving the resulting systems of linear equations. Determination at each time step of the values of the three integral invariants [43, 44] of the SWE, that is. the total mass, the total energy and the potential enstrophy, proved that the two-stage Numerov-Galerkin is attaining a consistently higher accuracy than the single-stage finite-element method (see Navon (1983) [45]).

In several seminal papers, Arakawa $[46,47]$ indicated that the integral constraints on quadratic quantities of physical importance, such as conservation of mean kinetic energy and mean square vorticity, will not be maintained in finite difference analogues of the equation of motion for twodimensional incompressible flow, unless the finite difference Jacobian expression for the advection term is restricted to a form that properly represents the interaction between grid points (i.e. use of staggered $\mathrm{C}$ or $\mathrm{D}$ grids).

Thus, the effect of conservation of integral invariants by finite-element discretization scheme of the SWE (3)-(5) as a measure of the correct discretization of long-term integrations has a pivotal importance. The numerical integration scheme is detailed in [35]. Using this program, we have captured the shallow-water dynamics over long-term numerical integrations (10-20 days). We will further detail the results in the section dedicated to numerical experiments. 


\section{DYNAMIC MODAL DECOMPOSITION OF FLOW FIELDS}

So far, we have noticed two directions in DMD technique. The straightforward approach is seeking a companion matrix that helps to construct in a least squares sense the final data vector as a linear combination of all previous data vectors [24, 48, 49]. Because this version may be ill-conditioned in practice, Schmid [50] recommends an alternate algorithm, based on averaging the mapping from the snapshots to the new one upon which the work within this article is based.

The aim of this section is twofold: first, we describe the classical analytical method for DMD. A DMD algorithm is then introduced as an improvement of the original algorithm, together with a criterion of optimal selection of the Koopman modes.

\subsection{The Koopman operator and the general description of dynamic mode decomposition}

Employing numerical simulations or experimental measurements techniques, different quantities associated with the flow are measured and collected as observations at one or more time signals, called observables. It turns out (see the survey of Bagheri [51]) that monitoring an observable over a very long time interval allows the reconstruction of the phase space.

Considering a dynamical system evolving on a manifold $\mathbb{M}$ such that, for all $w_{k} \in \mathbb{M}$

$$
w_{k+1}=f\left(w_{k}\right),
$$

the Koopman operator, defined by Koopman [25] in 1931, maps any scalar-valued function $g$ : $\mathbb{M} \rightarrow \mathbb{R}$ into a new function $U g$ given by

$$
U g(w)=g(f(w)) .
$$

The Koopman operator is infinite-dimensional and it steps forward in time an observable. Related to the spectral properties of the Koopman linear operator, the reader is invited to refer to Rowley et al. [24] for rigorous treatment on the subject. There is a unique expansion that expands each snapshot in terms of vector coefficients $\phi_{j}$, which are called Koopman modes and mode amplitudes $a_{j}(w)$, such that iterates of $w_{0}$ are then given by

$$
g\left(w_{k}\right)=\sum_{j=1}^{\infty} \lambda_{j}^{k} a_{j}\left(w_{0}\right) \phi_{j}, \quad \lambda_{j}=e^{\sigma_{j}+i \omega_{j}},
$$

where $\lambda_{j}$ are called the Ritz eigenvalues of the modal decomposition, which are complex-valued flow structures associated with the growth rate $\sigma_{j}$ and the frequency $\omega_{j}$.

Assuming that $\left\{w_{0}, w_{1}, \ldots w_{N}\right\}$ is a data sequence collected at a constant sampling time $\Delta t$, we define the following matrices

$$
V_{0}^{N-1}=\left(\begin{array}{llll}
w_{0} & w_{1} & \ldots & w_{N-1}
\end{array}\right), \quad V_{1}^{N}=\left(\begin{array}{llll}
w_{1} & w_{2} & \ldots & w_{N}
\end{array}\right) .
$$

The DMD algorithm is based on the hypothesis that a Koopman operator $\mathcal{A}$ exists, that steps forward in time the snapshots, such that

$$
w_{i+1}=\mathcal{A} w_{i}, \quad i=0, \ldots, N-1 .
$$

Schmid [50] assumed that the Koopman operator $\mathcal{A}$ is linear, but it was shown that the linearity assumption is not necessary in Rowley et al. [24]. It follows that the snapshots data set

$$
V_{0}^{N-1}=\left(\begin{array}{llll}
w_{0} & \mathcal{A} w_{0} & \mathcal{A}^{2} w_{0} \ldots \mathcal{A}^{N-1} w_{0}
\end{array}\right)
$$

corresponds to the $N^{t h}$ Krylov subspace generated by the Koopman operator from $w_{0}$.

Because the eigenvalues of the unknown matrix operator $\mathcal{A}$ must be obtained, a Galerkin projection of $\mathcal{A}$ onto the subspace spanned by the snapshots is performed. For a sufficiently long sequence of the snapshots, we suppose that the last snapshot $w_{N}$ can be written as a linear combination of the previous $N-1$ vectors, such that

$$
w_{N}=c_{0} w_{0}+c_{1} w_{1}+\ldots+c_{N-1} w_{N-1}+\mathcal{R}
$$


which can be written in matrix notation as

$$
w_{N}=V_{0}^{N-1} c+\mathcal{R} \mathrm{e}_{N-1}^{T},
$$

in which $c^{T}=\left(\begin{array}{llll}c_{0} & c_{1} \ldots c_{N-1}\end{array}\right)$ is a complex column vector and $\mathcal{R}$ is the residual vector. We assemble the following relations

$$
\mathcal{A}\left\{w_{0}, w_{1}, \ldots w_{N-1}\right\}=\left\{w_{1}, w_{2}, \ldots w_{N}\right\}=\left\{w_{1}, w_{2}, \ldots V_{0}^{N-1} c\right\}+\mathcal{R} \mathrm{e}_{N-1}^{T}
$$

in the matrix notation form,

$$
\mathcal{A} V_{0}^{N-1}=V_{1}^{N}=V_{0}^{N-1} \mathcal{C}+\mathcal{R e}_{N-1}^{T}, \quad \mathcal{C}=\left(\begin{array}{cccc}
0 & \ldots & 0 & c_{0} \\
1 & & 0 & c_{1} \\
\vdots & \vdots & \vdots & \vdots \\
0 & \ldots & 1 & c_{N-1}
\end{array}\right)
$$

where $\mathcal{C}$ is the companion matrix and $e_{j}^{T}$ represents the $j^{t h}$ Euclidean unitary vector of length $N-1$.

A direct consequence of (20) is that decreasing the residual increases the overall convergence and, therefore, the eigenvalues of the companion matrix $\mathcal{C}$ will converge toward some eigenvalues of the Koopman operator $\mathcal{A}$. Therefore, the way that we will monitor this convergence is by evaluating the size of the residual during the modal decomposition and plotting its $L_{2}$-norm.

Several methods have been employed so far to compute the companion matrix. The last column of the companion matrix may be found using the Moore-Penrose pseudoinverse [52] of $V_{0}^{N-1}$, as $c=\left(V_{0}^{N-1}\right)^{+} w_{N}=\left(\left(V_{0}^{N-1}\right)^{*} V_{0}^{N-1}\right)^{-1}\left(V_{0}^{N-1}\right)^{*} w_{N}$.

A solution for the linear least-square problem obtained from (20) is given by the economy size QR-decomposition of $V_{0}^{N-1}$, as it is discussed in [53].

Instead of using QR-decomposition, singular value decomposition (SVD) was also applied on $V_{0}^{N-1}$ in order to find the singular eigenvalues and vectors, this approach being helpful when the matrix $V_{0}^{N-1}$ is rank deficient [50]. The eigenelements of the companion matrix span the original data, and the decomposition (13) is achieved.

An error analysis of DMD [54, 55] proved that the procedure described earlier turns out to be ill-conditioned in practice. This may especially be the situation when modal decomposition is applied to snapshots collected at large time steps, which behave like using noisy experimental data, or for small time steps when the increments are smaller then the noise (aliasing). In this situation, the modal decomposition is unable to find proper eigenelements of the Koopman operator and the flow reconstruction is inaccurate. For these cases, we propose in the following an improved DMD algorithm.

\subsection{Description of an improved dynamic mode decomposition algorithm}

In this section, we consider that dynamical system (11) represents an approximation of the SWE model (3)-(5), discretized in both time and space, and the observable $w_{i}=w\left(t_{i}\right), t_{i}=i \Delta t$, $i=0, \ldots, N$, consist of the time dependent variables $w=\{u, v, h\}(x, y, t)$ in the spatial domain $\Omega$. The main objective is to find a representation of the flow field in the form

$$
w^{D M D}(x, y, t)=W_{b}+\sum_{j=1}^{r} a_{j} e^{\left(\sigma_{j}+i \omega_{j}\right) t} \phi_{j}(x, y), \quad \sigma_{j}=\frac{\log \left(\left|\lambda_{j}\right|\right)}{\Delta t}, \quad \omega_{j}=\frac{\arg \left(\left|\lambda_{j}\right|\right)}{\Delta t},
$$

where $\phi_{j} \in \mathbb{C}$ are the DMD modes, $r$ is the number of the DMD modes kept for flow decomposition, $a_{j} \in \mathbb{C}$ are the amplitudes of the modes, $\lambda_{j} \in \mathbb{C}$ are the Ritz eigenvalues of the modal decomposition associated with the growth rate $\sigma_{j}$ and the frequency $\omega_{j}$ and $W_{b}$ is a constant offset that represents the data mean, usually called the base flow in hydrodynamic stability analysis, 


$$
W_{b}(x, y)=\frac{1}{1+N} \sum_{i=0}^{N} w_{i}(x, y) .
$$

Theoretically, we apply the DMD on the mean-subtracted data $w_{i}^{\prime}=w_{i}-W_{b}, i=0, \ldots, N$. As noticed by Noack et al. [56], the use of the mean of a data set as the base flow represents a common practice in application of modal decomposition like POD. Recently, Chen, Tu and Rowley [57] pointed out that constructing the Koopman modes from base-flow-subtracted data offers the advantage that the ROM will satisfy the same boundary conditions employed for the full model, while computing DMD modes without first subtracting a base flow results in the boundary conditions not being satisfied.

The quantitative capabilities of DMD have already been well demonstrated in the literature by the efforts of Bagheri [22], Mezic [23], Rowley et al. [24] and Belson et al. [58]. The method of snapshots formulation is well-suited for large data because the eigenvalue problem does not depend on the dimension of the snapshot vector (see Holmes et al. [59]).

Here, we apply the method of snapshots introduced by Sirovich [60] in 1987 and we solve the resulting eigenvalue problem by a matrix multiplication method. We introduce in this paper a DMDbased approach yielding a supplementary subroutine for extracting the optimal Koopman modes. We summarize in the succeeding section the steps of the algorithm.

Algorithm 1: Improved dynamic mode decomposition for 2D flows with selection of dominant modes

(i) Collect data $w_{i}(x, y)=w\left(x, y, t_{i}\right), t_{i}=i \Delta t, i=0, \ldots, N$ from the flow field, equally distributed in time.

(ii) Placing the columns one after another, transform snapshots $w_{i}$ into columns $\widetilde{w}_{i}$ of the matrix

$$
\mathcal{V}=\left[\begin{array}{llll}
\widetilde{w}_{0} & \widetilde{w}_{1} & \ldots & \widetilde{w}_{N}
\end{array}\right]
$$

(iii) Compute the mean column $\bar{W}_{b}=\frac{1}{1+N} \sum_{i=0}^{N} \widetilde{w}_{i}$ and the mean-subtracted snapshot matrix $\mathcal{V}^{\prime}=$ $\mathcal{V}-\bar{W}_{b}$. Reshaping $\bar{W}_{b}$ into the matrix form corresponds to the base flow $W_{b}(x, y)$.

(iv) A matrix $V_{0}^{N-1}$ is formed with the first $N$ columns and the matrix $V_{1}^{N}$ contains the last $N$ columns of $\mathcal{V}^{\prime}$,

$$
\begin{aligned}
V_{0}{ }^{N-1} & =\left[\begin{array}{llll}
\widetilde{w}_{0} & \widetilde{w}_{1} & \ldots & \widetilde{w}_{N-1}
\end{array}\right], \\
V_{1}{ }^{N} & =\left[\begin{array}{lllll}
\widetilde{w}_{1} & \widetilde{w}_{2} & \ldots & \widetilde{w}_{N}
\end{array}\right] .
\end{aligned}
$$

Performing a Galerkin projection of the unknown Koopman operator $\mathcal{A}$ onto the subspace spanned by the snapshots, we express the vectors of $V_{1}^{N}$ as a linear combination of the independent sequence $V_{0}^{N-1}$ :

$$
V_{1}^{N}=\mathcal{A} V_{0}^{N-1}=V_{0}^{N-1} \mathcal{S}+\mathcal{R},
$$

where $\mathcal{R}$ is the residual matrix and $\mathcal{S}$ approximates the eigenvalues of $\mathcal{A}$ when the norm $\|\mathcal{R}\|_{2} \rightarrow 0$. The objective at this step is to solve the minimization problem

$$
\text { Minimize } \mathcal{\mathcal { S }}=\left\|V_{1}^{N}-V_{0}^{N-1} \mathcal{S}\right\| .
$$

(v) We identify a singular value decomposition of $V_{0}^{N-1}$ :

$$
V_{0}^{N-1}=U \Sigma W^{H},
$$


where $U$ contains the proper orthogonal modes of $V_{0}^{N-1}, \Sigma$ is a square diagonal matrix containing the singular values of $V_{0}^{N-1}$ and $W^{H}$ is the conjugate transpose of $W$. It follows from (26) that $\mathcal{S}$ can be obtained by multiplying $V_{1}^{N}$ by the Moore-Penrose pseudoinverse of $V_{0}^{N-1}$ :

$$
\mathcal{S}=\left(V_{0}^{N-1}\right)^{+} V_{1}^{N}=W \Sigma^{+} U^{H} V_{1}^{N}=X \Lambda X^{-1},
$$

where $X$ and $\Lambda$ represent the eigenvectors, respectively the eigenvalues of $\mathcal{S}$, and $\Sigma^{+}$is computed according to Moore-Penrose pseudoinverse definition of Golub and van Loan [52]:

$$
\Sigma^{+}=\operatorname{diag}\left(\frac{1}{\sigma_{1}}, \cdots, \frac{1}{\sigma_{r}}, 0 \cdots, 0\right), \quad r=\operatorname{rank}\left(V_{0}^{N-1}\right) .
$$

(vi) Calculation of Koopman modes and amplitudes. After solving the eigenvalue problem

$$
\mathcal{S} X=X \Lambda,
$$

the diagonal entries of $\Lambda$ represent the Ritz eigenvalues $\lambda$. The frequency and damping are provided by these eigenvalues. The projection of $V_{1}^{N}$ on the modes $V_{0}^{N-1} X$ yields:

$$
V_{1}^{N}=\left(V_{0}^{N-1} X\right) \Lambda X^{-1}=\mathcal{A} V_{0}^{N-1} .
$$

From this expression, the contribution of each dynamic mode to the data sequence $V_{1}^{N}$ is obtained. Thus, the Koopman modes are the columns of the matrix $\phi=V_{0}^{N-1} X$. Reshape these columns back into matrix form to obtain the dynamic modes $\phi_{i}(x, y)=\phi\left(x, y, t_{i}\right)$, $t_{i}=i \Delta t, i=0, \ldots, N-1$. The amplitudes are given by the norm of the corresponding column vector of $V_{0}^{N-1} X$, as

$$
a_{j}=\frac{\left\|V_{0}^{N-1} X(:, j)\right\|_{2}}{\left\|V_{0}^{N-1} X\right\|_{2}}, \quad j=1, \ldots, r,
$$

where $r$ represents the number of the Koopman modes stored for modal decomposition.

A novel and efficient technique to select the dominant Koopman modes will be given in the following.

\subsection{Optimal selection of the dominant Koopman modes}

Selection of Koopman modes and amplitudes used for the flow reconstruction constitutes the source of many discussions among modal decomposition practitioners. For instance, Jovanovic et al. [61] introduced a low-rank DMD algorithm to identify an a priori specified number of modes that provide optimal approximation of experimental or numerical snapshots at a certain time interval. Consequently, the modes and frequencies that have the strongest influence on the quality of approximation have been selected. Chen et al. [57] introduced an optimized DMD, which tailors the decomposition to an optimal number of modes. This method minimizes the total residual over all data vectors and uses simulated annealing and quasi-Newton minimization iterative methods for selecting the optimal frequencies.

The superposition of all Koopman modes, weighted by their amplitudes and complex frequencies, approximates the entire data sequence, as relation (21) describes, but there are also modes that have a weak contribution. We address in this section the problem of identification of an optimal truncated representation of the flow field in order to capture the most important dynamic structures.

To this end, we seek for a number $r_{D M D}<r$, which represents the optimal number of the selected modes that must be identified, such that the flow can be reconstructed using the first $r_{D M D}$ optimal Koopman modes and associated amplitudes and Ritz eigenvalues as:

$$
w^{D M D}(x, y, t)=W_{b}(x, y)+\sum_{j=1}^{r_{D M D}} a_{j} \lambda_{j} \phi_{j}(x, y) .
$$


In matrix formulation, relation (34) yields:

$$
w^{D M D}(x, y, t)=W_{b}+\left(\begin{array}{llll}
a_{1} & a_{2} & \ldots & a_{r_{D M D}}
\end{array}\right)\left(\begin{array}{ccc}
\lambda_{1} & \ldots & 0 \\
\vdots & \vdots & \vdots \\
0 & \ldots & \lambda_{r_{D M D}}
\end{array}\right)\left(\begin{array}{c}
\phi_{1}(x, y) \\
\vdots \\
\phi_{r_{D M D}}(x, y)
\end{array}\right) .
$$

In POD or POD Balanced Truncation Method [49], the flow field is decomposed into orthogonal modes, which are by construction ranked by energy level through the POD or Hankel eigenvalues [51]. Thereafter, the order of the modes is in decreasing amount of energy, and the POD modes are designed to contain the largest amount of energy with any given number of modes. In DMD, the modes are not orthogonal, but one advantage of DMD compared with POD is that each DMD mode is associated with a pulsation and a growth rate, and each mode has a single distinct frequency. For this feature, the DMD method was originally used in nonlinear dynamics, for instance Muld et al. [29], and was just recently introduced in fluid mechanics [28, 62, 63].

A criterion of selecting the DMD modes can be their amplitude, $a_{j}$, or based on their frequency/growth rate, $\omega_{j} / \sigma_{j}$. As reported by Noack et al. [64], the amplitude criterion is not sufficient because there exist modes that are very rapidly damped, having very high amplitudes. The modal selection based on frequency/growth rate is not rigorous because it relies on a priori physical knowledge of the flow. The frequencies resolved by the DMD are still subjected to the Nyquist sampling theorem [65], and the researcher has to know in advance, frequencies that are essential in the flow physics to adjust the sampling interval $\Delta t$. On the other hand, the non-orthogonality of the Koopman modes may raise the projection error while increasing the order of the DMD basis.

To avoid these difficulties, we introduce in the following a new method to optimize the selection of the Koopman modes involved in the reconstruction of the flow. The DMD algorithm that we propose is based on the conservation of quadratic integral invariants by the finite-element discretization scheme of the shallow-water model (3)-(5). We assume that the reduced order reconstructed flow (34) also preserves the conservation of the total flow energy. In parallel, we aim to eliminate the modes that contribute weakly to the data sequence. Let

$$
E=\frac{1}{N+1} \sum_{i=0}^{N} \iint_{\Omega} h_{i}(x, y)\left(u_{i}(x, y)^{2}+v_{i}(x, y)^{2}\right)+g h_{i}(x, y)^{2} d x d y,
$$

be the total energy of the high fidelity flow, defined also in [43], or in Hamiltonian form in [66] and

$$
\begin{aligned}
E^{D M D}= & \frac{1}{N+1} \sum_{i=0}^{N} \iint_{\Omega} h_{i}^{D M D}(x, y)\left(u_{i}^{D M D}(x, y)^{2}+v_{i}^{D M D}(x, y)^{2}\right) \\
& +g h_{i}{ }^{D M D}(x, y)^{2} d x d y,
\end{aligned}
$$

be the total energy of the reduced order flow, where $\left(h_{i}, u_{i}, v_{i}\right)(x, y)$ and $\left(h_{i}{ }^{D M D}\right.$, $\left.u_{i}{ }^{D M D}, v_{i}^{D M D}\right)(x, y), i=0, \ldots, N$ represents the full rank flow, respectively, the Koopman decomposed flow at time $i$.

We arrange the Koopman modes in descending order of the energy of the modes, weighted by the inverse of the Strouhal number $S t=\arg \left(\lambda_{j}\right) /(2 \pi \Delta t)$ :

$$
e_{j}^{D M D}=\frac{1}{S t} \cdot \frac{\left\|\phi_{j}(x, y)\right\|_{F}}{\|\mathcal{V}\|_{F}}, \quad j=1, \ldots, r .
$$

We denote by $\|\cdot\|_{F}$ the Frobenius matrix norm in the sense that for any matrix $A \in \mathbb{C}_{m \times n}$ having singular values $\sigma_{1}, \ldots, \sigma_{n}$ and SVD of the form $A=U \Sigma V^{H}$, then

$$
\|A\|_{F}=\left\|U^{H} A V\right\|_{F}=\|\Sigma\|_{F}=\sqrt{\sigma_{1}^{2}+\ldots+\sigma_{n}^{2}} .
$$


Determination of the optimal vector of amplitudes and corresponding eigenvalues and Koopman modes $\left(a_{j}, \lambda_{j}, \phi_{j}(x, y)\right), j=1, \ldots, r_{D M D}$ then amounts to finding the solution to the following optimization problem

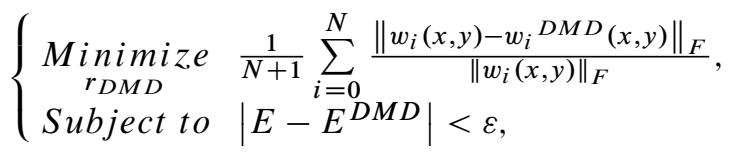

where $w_{i}(x, y)$ and $w_{i}{ }^{D M D}(x, y), i=0, \ldots, N$ represents the full rank flow, respectively, the Koopman decomposed flow at time $i$ and $\varepsilon=10^{-5}$ sets an upper bound on the relative error because of rounding in floating point arithmetic.

We are interested in finding the Koopman modes that provide the maximum energy of the fluctuations at distinct frequencies and we continue minimizing the residual subject to the linear dynamics constraint and the flow energy conservation assumption.

\section{DECOMPOSITION OF FLOW FIELDS BY PROPER ORTHOGONAL DECOMPOSITION SUBJECT TO THE LINEAR DYNAMICS}

Apart from Krylov subspace methods, the POD represents at the moment state-of-the-art for many model reduction problems. The strong point of POD is that it can be applied to nonlinear partial differential equations, especially for smooth systems in which the energetics can be characterized by the first few modes. The applicability of POD to complex systems is limited mainly because of errors associated with the truncation of POD modes. The POD and its variants are also known as Karhunen-Loeve expansions in feature selection and signal processing, empirical orthogonal functions in atmospheric science or principal component analysis in statistics. In weather and climate modelling [7, 12, 13, 33], as well as in other complex systems such as data assimilation $[15,16]$, the development of accurate and reliable low-dimensional models represents an extremely important task.

The idea underlying this method is that the time response of a system, given a certain input, contains the essential behavior of the system. Therefore, the set of outputs serves as a starting-point for POD. We consider that the observables $w_{i}=w\left(t_{i}\right), t_{i}=i \Delta t, i=0, \ldots, N$, consist of the time dependent variables $w=\{u, v, h\}(x, y, t)$ of the SWE model (3)-(5), discretized in both time and space in the spatial domain $\Omega$. The main objective in POD is to find a representation of the flow field of the form

$$
w^{P O D}(x, y, t)=W_{b}(x, y)+\sum_{j=1}^{r_{P O D}} b_{j}(t) \Phi_{j}(x, y),
$$

where $W_{b}(x, y)$ represents the data mean flow defined as in (22), $r_{P O D}$ represents the optimal number of the POD selected modes that must be identified. The time dependent coefficients $b_{j}$ are called Fourier coefficients. We are looking for an orthonormal basis $\left\{\Phi_{j}(x, y)\right\}, j=1, \ldots, r_{P O D}$ such that the averages of the first few Fourier-coefficients represent $99 \%$ of the total energy of the snapshots. This leads to the following POD algorithm:

Algorithm 2: Proper orthogonal decomposition algorithm for 2D flows

(i) Collect data $w_{i}(x, y)=w\left(x, y, t_{i}\right), t_{i}=i \Delta t, i=0, \ldots, N$ from the flow field, equally distributed in time.

(ii) Placing the columns one after another, transform snapshots $w_{i}$ into columns $\widetilde{w}_{i}$ of the matrix

$$
\mathcal{V}=\left[\begin{array}{llll}
\widetilde{w}_{0} & \widetilde{w}_{1} & \ldots & \widetilde{w}_{N}
\end{array}\right]
$$

(iii) Compute the mean column $\bar{W}_{b}=\frac{1}{N+1} \sum_{i=0}^{N} \widetilde{w}_{i}$ and the mean-subtracted snapshot matrix $\mathcal{V}^{\prime}=$ $\mathcal{V}-\bar{W}_{b}$. Reshaping $\bar{W}_{b}$ into the matrix form corresponds to the base flow $W_{b}(x, y)$. 
(iv) Calculate the empirical correlation matrix

$$
C=\frac{1}{N+1} \mathcal{V}^{\prime} \mathcal{V}^{\prime T}
$$

where $N+1$ represents the number of snapshots and $\mathcal{V}^{\prime T}$ represents the transpose of the mean subtracted snapshot matrix.

(v) Compute the eigenvalue decomposition

$$
C v_{j}=\lambda_{j} v_{j}, \quad j=1, \ldots, N+1, \quad v_{j} \in \mathbb{R}^{N+1},
$$

where $N+1$ represents the number of the total eigenvalues.

(vi) Find the number of POD basis vectors $r_{P O D}$ capturing $99.99 \%$ of the snapshots energy, defined as

$$
e_{P O D}=\sum_{j=1}^{r_{P O D}} \lambda_{j} / \sum_{j=1}^{N+1} \lambda_{j} .
$$

(vii) We can choose the first orthonormal basis of eigenvectors $\left\{v_{1}, \ldots, v_{r_{P O D}}\right\}$, and the corresponding POD basis functions are given by

$$
\Phi_{j}=\frac{1}{\sqrt{\lambda_{j}}} \mathcal{V}^{\prime} v_{j}, \quad j=1, \ldots, r_{P O D}
$$

(viii) The temporal coefficients are stored in the matrix $B$, which is obtained by relation

$$
B=\Phi^{T} \mathcal{V}^{\prime}
$$

Hence, in each row we find the trajectories of the dynamical system at discrete time events.

(ix) The reconstruction of the flow fluctuating part is achieved as $\mathcal{V}_{P O D}^{\prime}=\Phi B$, and the reconstruction of the flow field is $\mathcal{V}_{P O D}=\mathcal{V}_{P O D}^{\prime}+\bar{W}_{b}$. Reshape the columns of $\mathcal{V}_{P O D}$ back into matrix form to obtain the POD representation of the flow $w^{P O D}(x, y, t)$.

\section{ANALYSIS OF SHALLOW WATER EQUATIONS COHERENT STRUCTURES BY KOOPMAN MODES AND PROPER ORTHOGONAL DECOMPOSITION}

We perform the numerical experiments in a rectangular channel whose dimensions are $D_{\max }=$ $4400 \mathrm{~km}, L_{\max }=6000 \mathrm{~km}$. The dimensional constants used for the model are

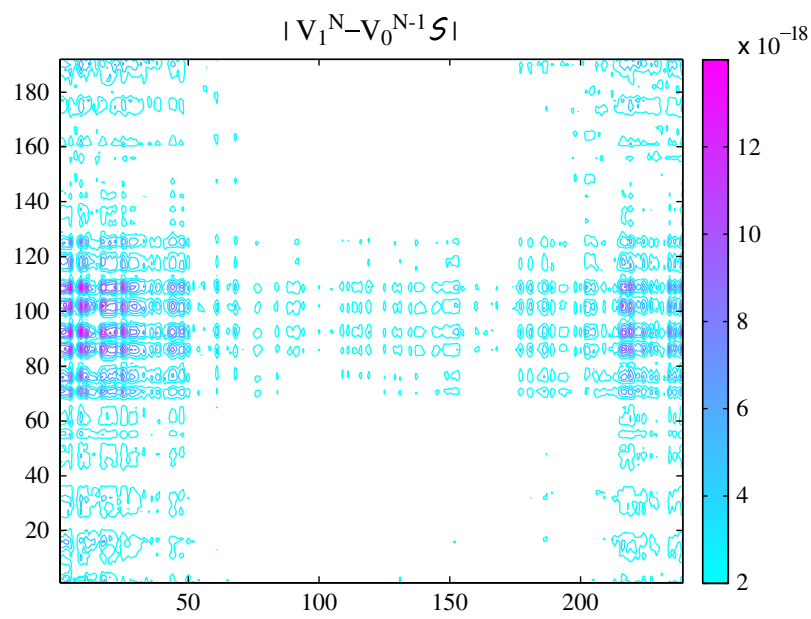

Figure 1. The error of the minimization problem (27) in the computation of the geopotential height field. 


$$
\begin{aligned}
& \widehat{f}=10^{-4} s^{-1}, \quad \beta=1.5 \times 10^{-11} s^{-1} m^{-1}, \quad g=10 m s^{-1}, \\
& H_{0}=2000 \mathrm{~m}, \quad H_{1}=220 \mathrm{~m}, \quad H_{2}=133 \mathrm{~m} \text {. }
\end{aligned}
$$

In this section, the application of POD and DMD is illustrated by comparing the evolution of the flow field along the integration time window. There are several major differences between these two decomposition methods. The spatial basis functions $\phi_{j}(x, y)$ and $\Phi_{j}(x, y)$, for DMD and POD, respectively, offer an insight into the coherent structures in the flow field. The differences between $\phi_{j}(x, y)$ and $\Phi_{j}(x, y)$ occur because of the principles of the decomposition methods. The time evolution of a DMD mode is influenced by the multiplication of the complex eigenvalue $\lambda_{j}$ of the Koopman operator weighted by the amplitude, while the time evolution of POD modes is described

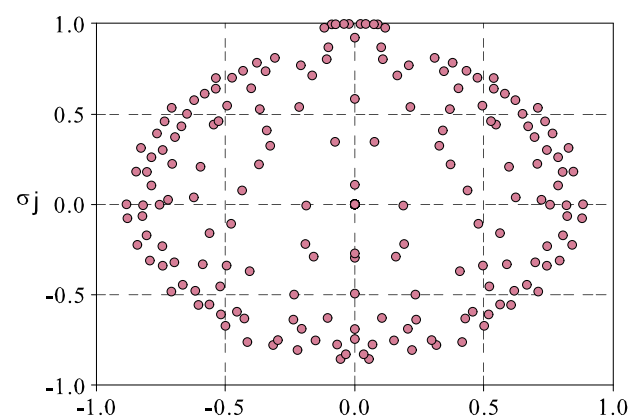

a.

$\omega$

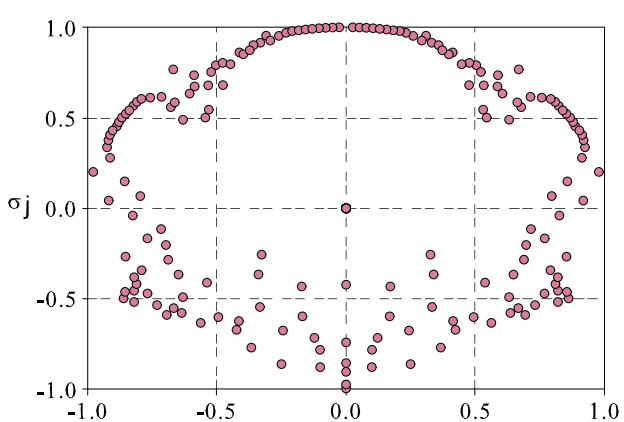

b.

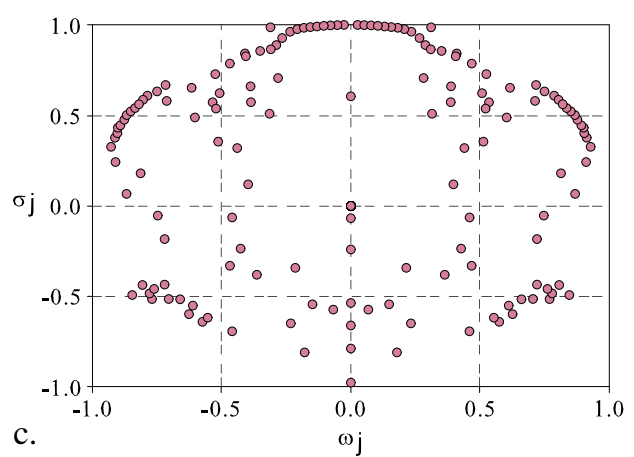

Figure 2. Spectrum of the dynamic mode decomposition: (a) geopotential field $h$; (b) streamwise velocity field $u$; and (c) spanwise velocity field $v, \Delta t=600 \mathrm{~s}$.
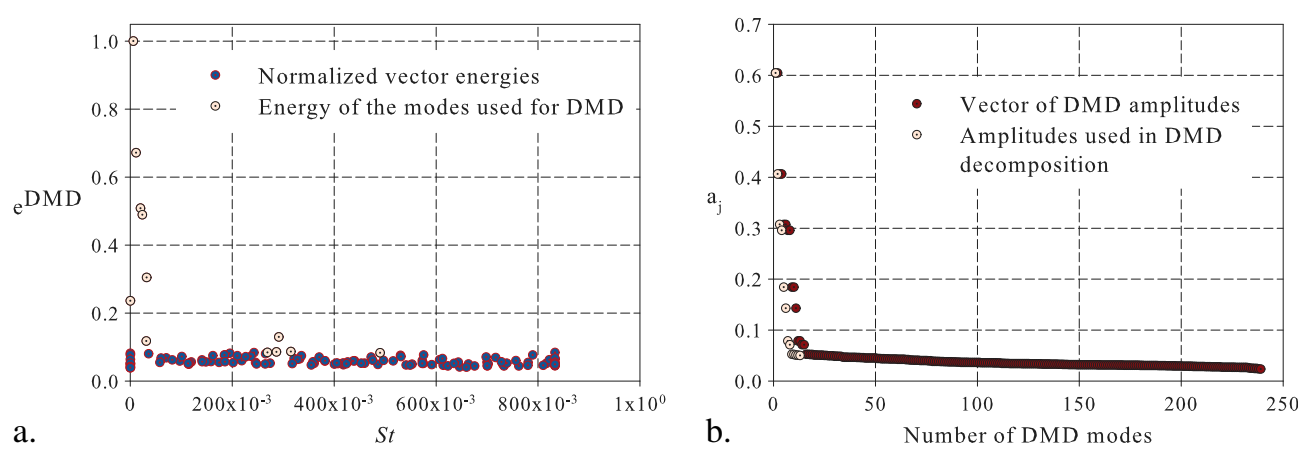

Figure 3. Decomposition of geopotential height field $h$ using the improved dynamic mode decomposition (DMD) algorithm: The lighter colored dots indicate the modes for which the amplitude values and Ritz eigenvalues are kept in the flow reconstruction. (a) the normalized vector energy versus the Strouhal number; and (b) the amplitudes of the DMD modes, sorted in descending order. 
by the functions $b_{j}(t)$. The POD modes are orthonormal in space with the energy inner product. In DMD, each mode oscillates at a single frequency, hence the expression that the DMD modes are orthogonal in time.

\subsection{Numerical results for the improved dynamic mode decomposition algorithm}

As an improvement of the classic algorithm of Schmid [50], we explore the subtraction of the data mean of the flow when DMD algorithm is applied to large time step observation. Unlike the classic algorithm, we arrange the Koopman modes in descending order of the energy of the DMD modes weighted by the inverse of the Strouhal number, defined by relation (38). In addition, the novelty introduced in this paper resides in the selection of the DMD modes and associated amplitudes and Ritz values as a solution of the constrained optimization problem (40).

In order to assess the performances of the method proposed, we have considered two numerical experiments. In the first experiment, we record a number of 240 unsteady solutions of the twodimensional SWE model (3)-(5), with time step $\Delta t=600 \mathrm{~s}$. Figure 1 presents the error of the minimization problem (27), which confirms that the use of the Moore-Penrose inverse in the DMD computation produces an accurate result.

The DMD spectra for the mean-subtracted fields $(u, v, h)(x, y, t)$ are presented in Figure 2. The improved DMD technique presented herein is fully capable of determining the modal growth rates and the associated frequencies.

Figure 3a presents the normalized vector energy (38) versus the Strouhal number. The lighter colored dots indicate the modes for which the corresponding amplitudes and Ritz eigenvalues are
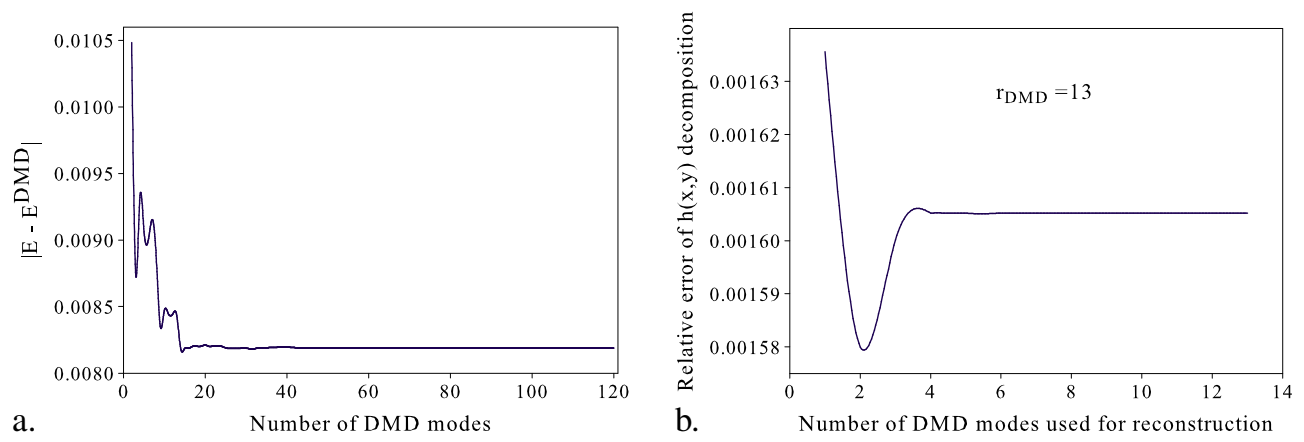

Figure 4. Improved dynamic mode decomposition (DMD) algorithm: (a) absolute error between the total energy of the high fidelity flow and the total energy of the reduced order flow, as the number of the DMD modes; and (b) The relative error $\frac{\left\|h(x, y)-h^{D M D}(x, y)\right\|_{F}}{\|h(x, y)\|_{F}}$ of geopotential height field decomposition, using $r_{D M D}=13$ modes.
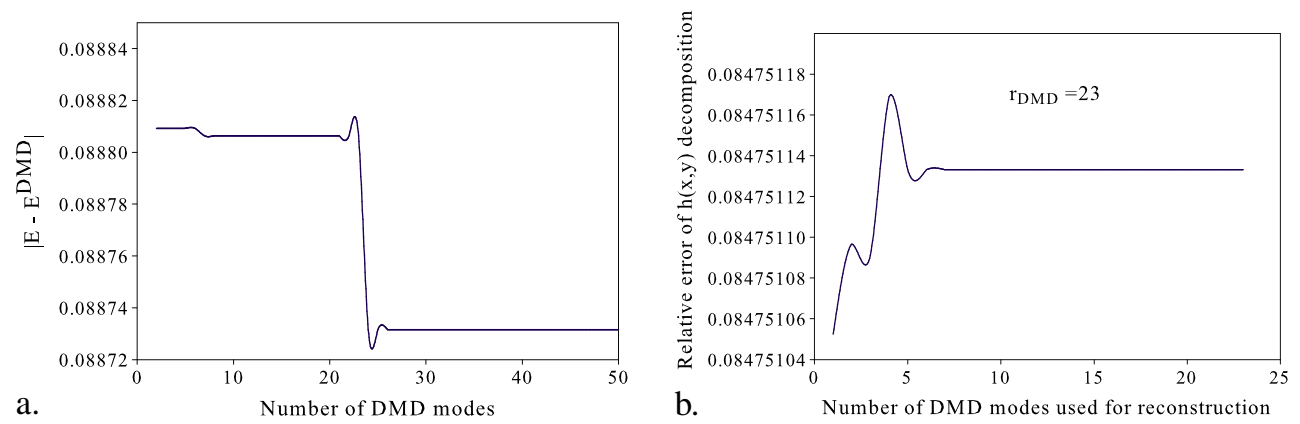

Figure 5. Classic dynamic mode decomposition (DMD) algorithm: (a) absolute error between the total energy of the high fidelity flow and the total energy of the reduced order flow, as the number of the DMD modes; and (b) the relative error $\frac{\left\|h(x, y)-h^{D M D}(x, y)\right\|_{F}}{\|h(x, y)\|_{F}}$ of geopotential height field decomposition, using $r_{D M D}=23$ modes. 
kept in the flow reconstruction. Figure $3 \mathrm{~b}$ shows that the higher amplitudes are associated to the most energetic Koopman modes selected for flow decomposition. This demonstrates that in DMD decomposition, the amplitudes are directly proportional to the energy in the coherent structures, as defined in Equation (38), unlike the POD decomposition where the eigenvalues capturing most of the snapshots energy indicate the corresponding POD basis functions.

A novel selection method for the DMD modes has been proposed in this paper, based on the conservation of total flow energy by the finite-element discretization scheme of the shallow-water model. We have computed the double integral representing the flow energy defined, respectively, in Equations (36) and (37) using Simpson's 1/3 rule, considering DMD decomposition with an incremental number of modes, and we solved the constrained optimization problem (40) employing the sequential quadratic programming (SQP) [67]. Solution of the constrained optimization problem (40) leads to the number of $r_{D M D}=13$ Koopman modes and associated amplitudes and Ritz values to be used in flow decomposition in the case of the improved DMD algorithm, while a number of $r_{D M D}=23$ dominant Koopman modes were detected in the case of the classic DMD algorithm.

The absolute error between the total energy of the high fidelity flow and the total energy of the reduced order flow, that is, $\left|E-E^{D M D}\right|$, in case of application of the improved DMD algorithm is represented in Figure 4a. The relative error of geopotential height field decomposition, defined as $\frac{\left\|h(x, y)-h^{D M D}(x, y)\right\|_{F}}{\|h(x, y)\|_{F}}$ using $r_{D M D}=13$ modes is depicted in Figure $4 \mathrm{~b}$. The absolute error between the total energy of the high fidelity flow and the total energy of the reduced order flow, in case of application of the classic DMD algorithm, is represented in Figure 5a. The relative error of geopotential height field decomposition, using $r_{D M D}=23$ modes is depicted in Figure $5 \mathrm{~b}$. A
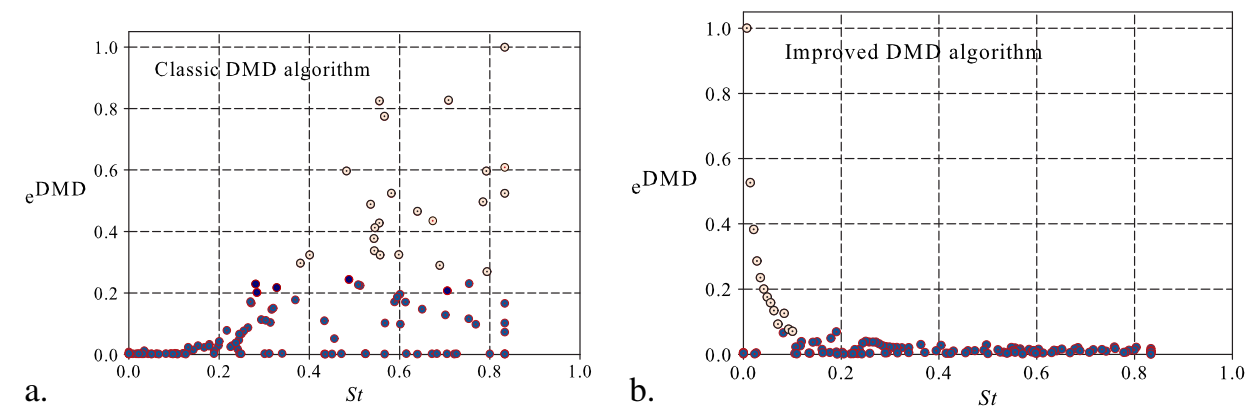

Figure 6. Decomposition of streamwise velocity field $u$-The normalized vector energy versus the Strouhal number: (a) application of classic dynamic mode decomposition (DMD) algorithm [50] and (b) application of improved DMD algorithm-present approach. The lighter colored dots indicate the amplitude values for which the corresponding modes and Ritz eigenvalues are kept in the flow reconstruction.
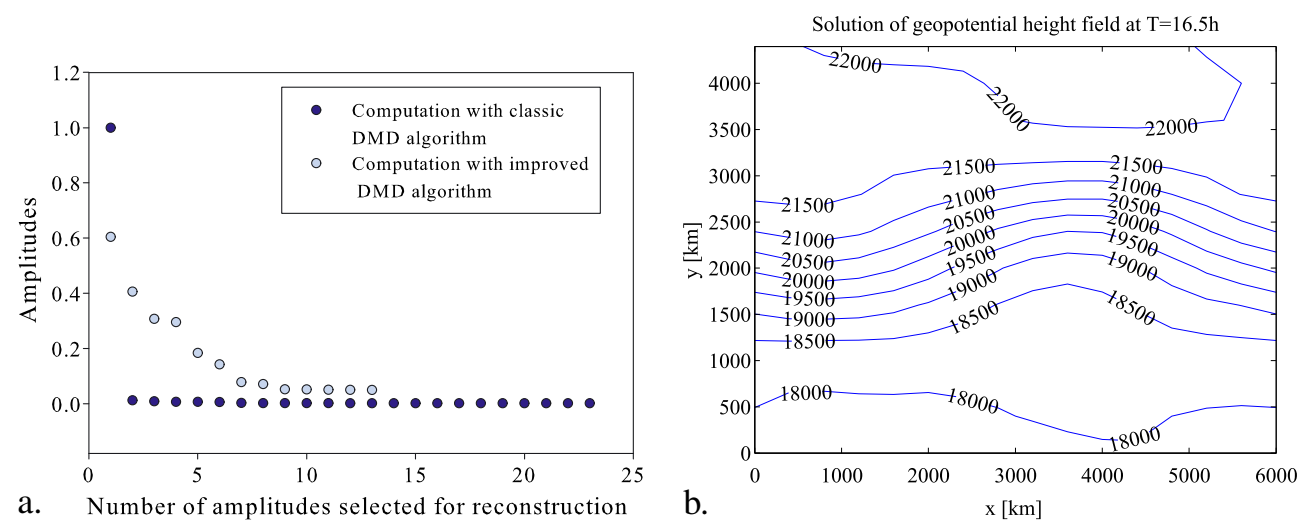

Figure 7. (a) Comparative analysis of the amplitudes used in reconstruction of the geopotential height field and (b) finite-element solution of the geopotential height field. 
comparative analysis of the presented results indicates that the improved DMD algorithm is more efficient in the flow reconstruction than the classic one.

A comparative representation of the energy of the modes versus the Strouhal number is presented in Figure 6, where the classic DMD algorithm [50] and the improved DMD algorithm presented in this paper have been applied for decomposition of the streamwise velocity field $u$. In the classic DMD decomposition, the modal energy exhibits a divergent tendency as the Strouhal number increases. In an opposite manner, in the improved DMD algorithm employed in the present research, the most energetic Koopman modes selected for flow decomposition correspond to low Strouhal numbers.

A comparative analysis of the amplitudes selected in reconstruction of the geopotential height field is illustrated in Figure 7a. It is obvious that decomposition computed with the classic DMD algorithm exhibits a higher amplitude and a series of lower amplitudes. Instead, the improved DMD
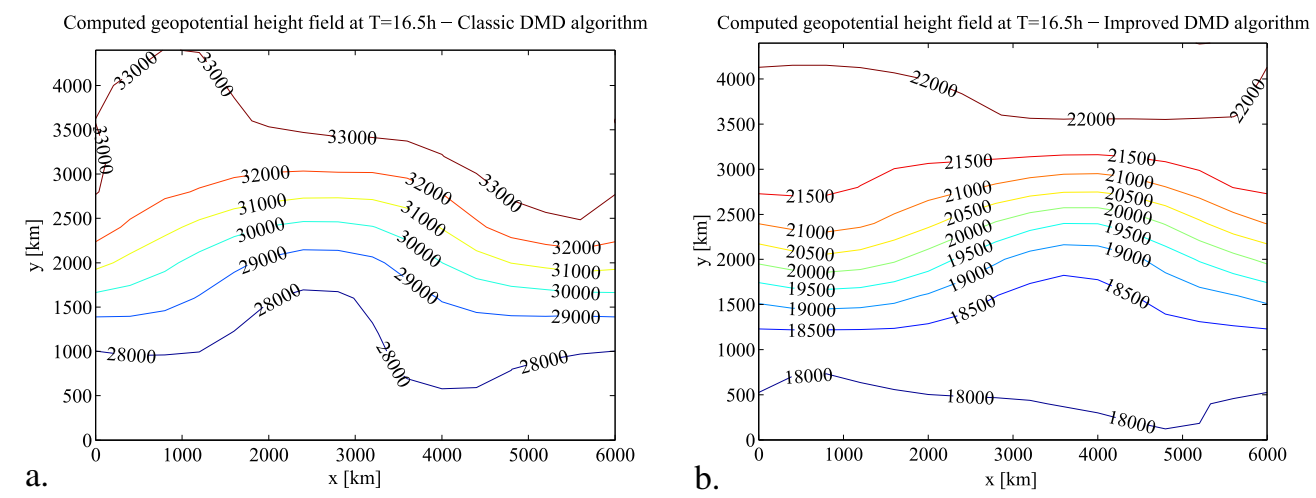

Figure 8. (a) Reconstruction of the geopotential height field at time $T=16.5 \mathrm{~h}$ employing classic dynamic mode decomposition (DMD) algorithm [50], $r_{D M D}=23$; (b) reconstruction of the geopotential height field at time $T=16.5 \mathrm{~h}$ employing improved DMD algorithm - present research, $\Delta t=600 \mathrm{~s}, r_{D M D}=13$.
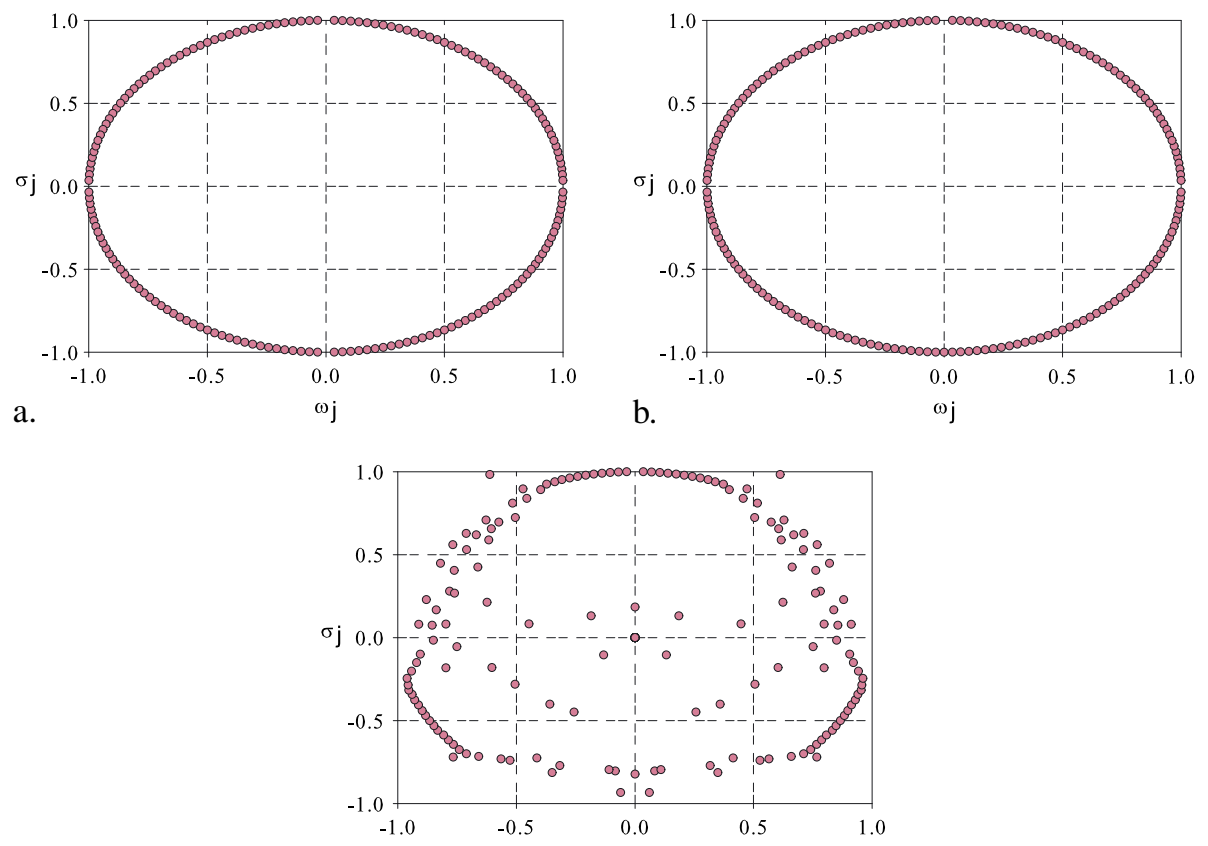

c.

$\omega_{\mathrm{j}}$

Figure 9. Spectrum of the dynamic mode decomposition: (a) geopotential field $h$; (b) streamwise velocity field $u$; and (c) spanwise velocity field $v, \Delta t=1200 s$. 
algorithm generates a normal distribution of the amplitudes. We perform the reconstruction of the geopotential height field at time $T=16.5 h$ in Figure 8, employing both the classic DMD and the improved DMD algorithm. Comparing the results with the finite-element solution depicted in Figure $7 \mathrm{~b}$, we conclude that the improved DMD algorithm leads to more accurate reconstruction than the classic method.

In the second experiment, we record a number of 180 unsteady solutions of the two-dimensional SWE model (3)-(5) and we double the time step at $\Delta t=1200 \mathrm{~s}$. We perform the geopotential
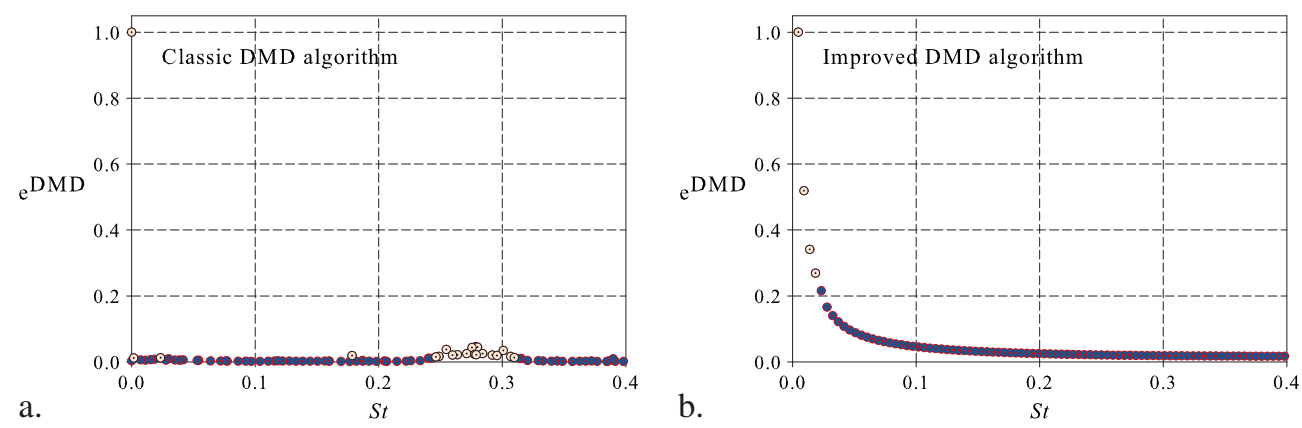

Figure 10. The normalized vector energy versus the Strouhal number: The lighter colored dots indicate the modes for which the amplitude values and Ritz eigenvalues are retained in the flow decomposition. (a) The classic dynamic mode decomposition (DMD) algorithm and (b) Improved DMD algorithm-present research, $\Delta t=1200$ s.
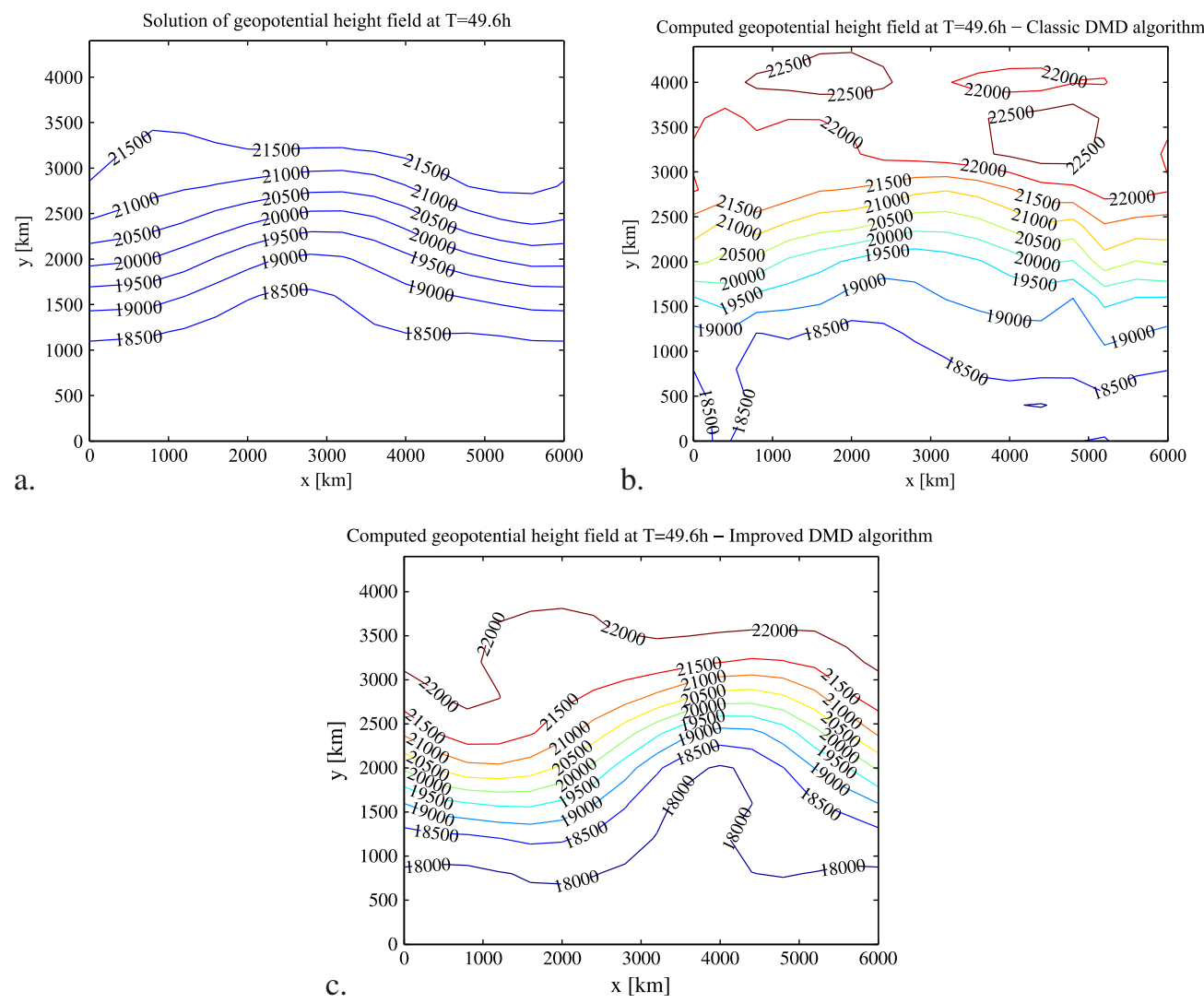

Figure 11. (a) Finite-element solution of the geopotential height field at time $T=49.6 h$. (b) Reconstruction of the geopotential height field at time $T=49.6 \mathrm{~h}$ employing classic dynamic mode decomposition (DMD) algorithm [50], $r_{D M D}=21$; (c) reconstruction of the geopotential height field at time $T=49.6 \mathrm{~h}$ employing improved DMD algorithm-present research, $\Delta t=1200 s, r_{D M D}=4$. 
height flow decomposition at time $T=49.6 \mathrm{~h}$. The DMD spectra for the mean-subtracted fields $(u, v, h)(x, y, t)$ are presented in Figure 9. It is easy to see that the Ritz values are the roots of unity in the case of geopotential field $h$ and streamwise velocity $u$ decompositions. This occurs in the situations where the minimization problem (27) produces a nonsingular matrix $\mathcal{S}$.

Solution of the constrained optimization problem (40) leads to the number of $r_{D M D}=21 \mathrm{Koop}-$ man modes in the case of the classic DMD algorithm, while a number of only $r_{D M D}=4$ Koopman modes are retained in the case of the improved DMD algorithm (the reader is referred to Figure 10).

Geopotential height field reconstruction at time $T=49.6 \mathrm{~h}$ is depicted in Figure 11, in comparison.

Analysing the numerical results, we are able to conclude that the improved DMD algorithm presented herein is more efficient in the reconstruction of large time step observation because an accurate solution is achieved with a much smaller number of modes than in the case of employment of classic DMD decomposition. In the next section, a qualitative comparison between the DMD and POD modes is given.

\subsection{Numerical results for proper orthogonal decomposition algorithm}

Considering the set of 180 snapshot used also in the previous investigation, representing unsteady solutions of the two-dimensional SWE model (3)-(5) computed with the time step $\Delta t=1200 \mathrm{~s}$, we employ the POD algorithm described in Section 4 to obtain the reconstruction of the geopotential height field at time $T=49.6 \mathrm{~h}$. We plot in Figure 12 the singular values obtained from POD
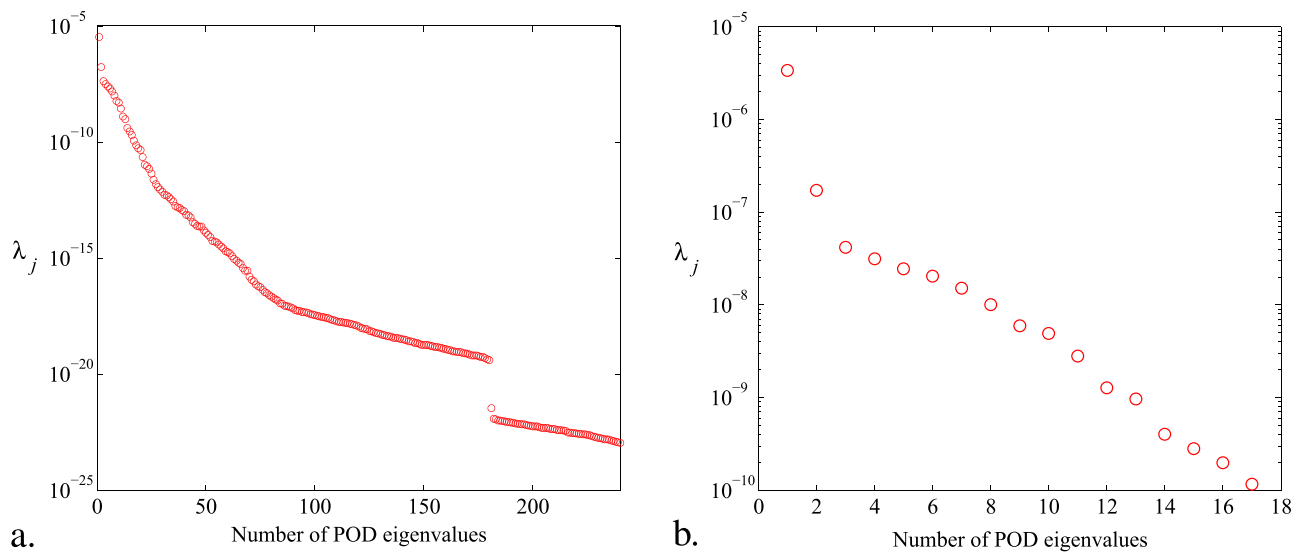

Figure 12. (a) Proper orthogonal decomposition (POD) eigenvalues and (b) based on an energetic criterion, $r_{P O D}=17$ modes are kept for the POD expansion.
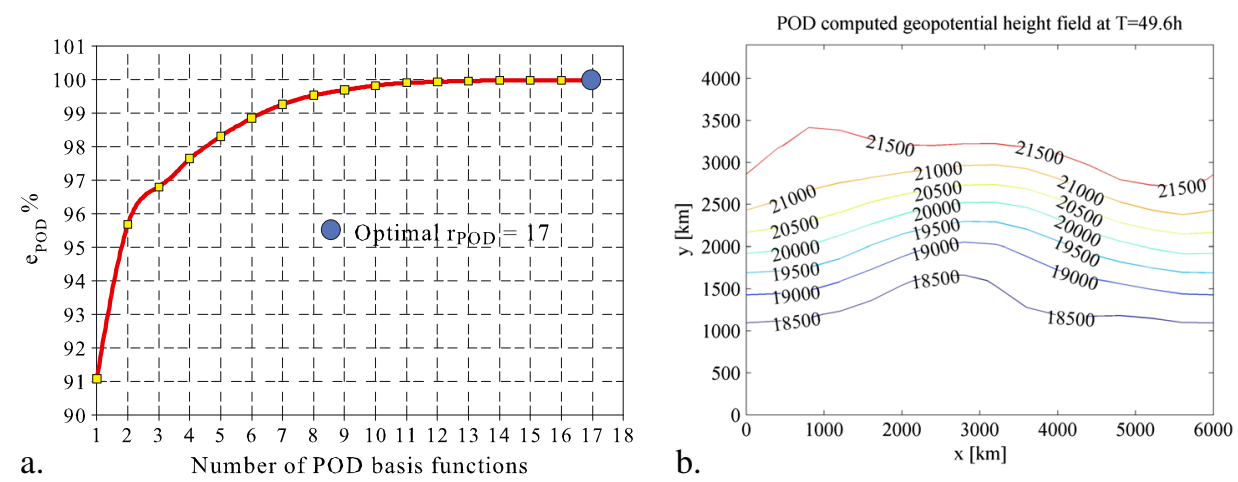

Figure 13. (a) The energy captured in the proper orthogonal decomposition (POD) as the number of the POD modes and (b) POD reconstruction of geopotential height field at time $T=49.6 h$, using $r_{P O D}=$ 17 modes. 
decomposition. Most of the energy defined in Equation (45) is contained in the first few modes. Specifically, the number of optimal POD basis functions is $r_{P O D}=17$, because the first 17 eigenvalues yield more than $99.99 \%$ of the snapshots energy (Figure 13a). Representation of the POD computed geopotential height field is presented in Figure 13b.

Comparing the flow decomposition by the improved DMD algorithm in Figure 11c with the POD decomposition in Figure 13b, we conclude that the flow reconstruction using the POD method is more accurate than reconstruction by the DMD method. Instead, DMD provides an acceptable reduced analytical linear model of considered nonlinear dynamical system.

The first four Koopman modes computed with the improved DMD algorithm presented herein are depicted in Figure 14, beside the first four POD basis functions, for modal decomposition of the geopotential height field.

A quantitative comparison of the spatial modes computed from the two decompositions discussed here can be obtained from the Modal Assurance Criterion (MAC), as recommended by Brown et al. [68]. The MAC is a measure of the degree of linearity between two vectors. The MAC value for a pair of modes is defined as

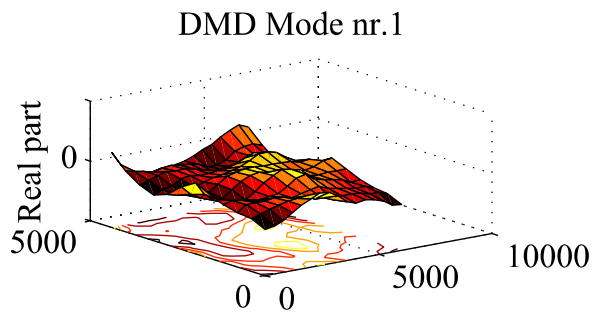

DMD Mode nr.2

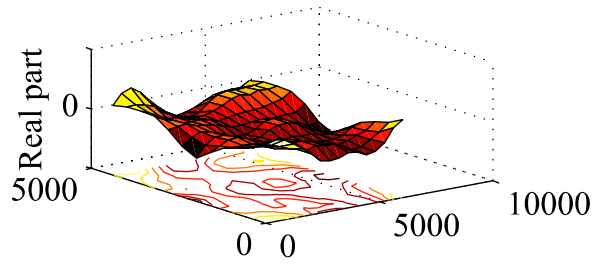

DMD Mode nr.3

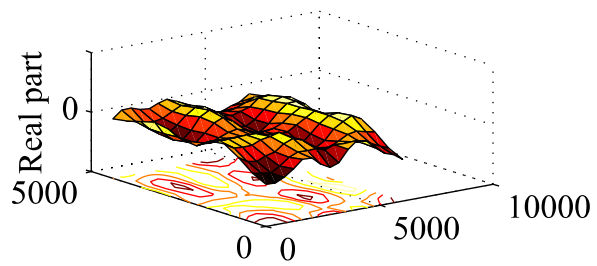

DMD Mode nr.4

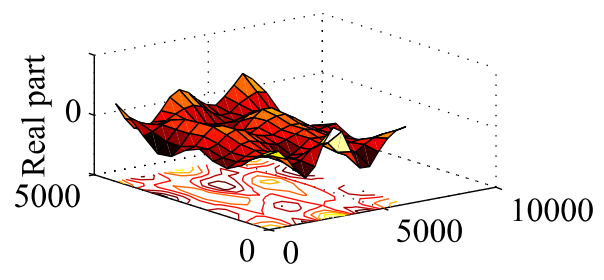

POD Mode nr.1

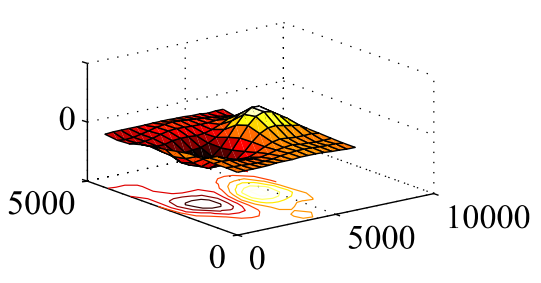

POD Mode nr.2

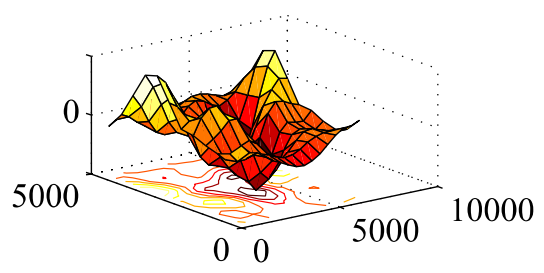

POD Mode nr.3

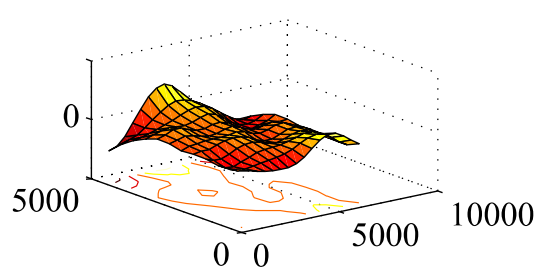

POD Mode nr.4

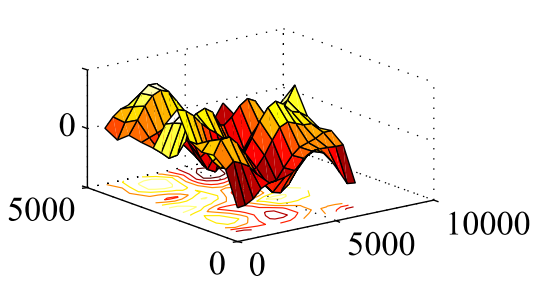

Figure 14. Left column: first four Koopman modes retained in dynamic mode decomposition. Right column: first four proper orthogonal decomposition basis functions. 

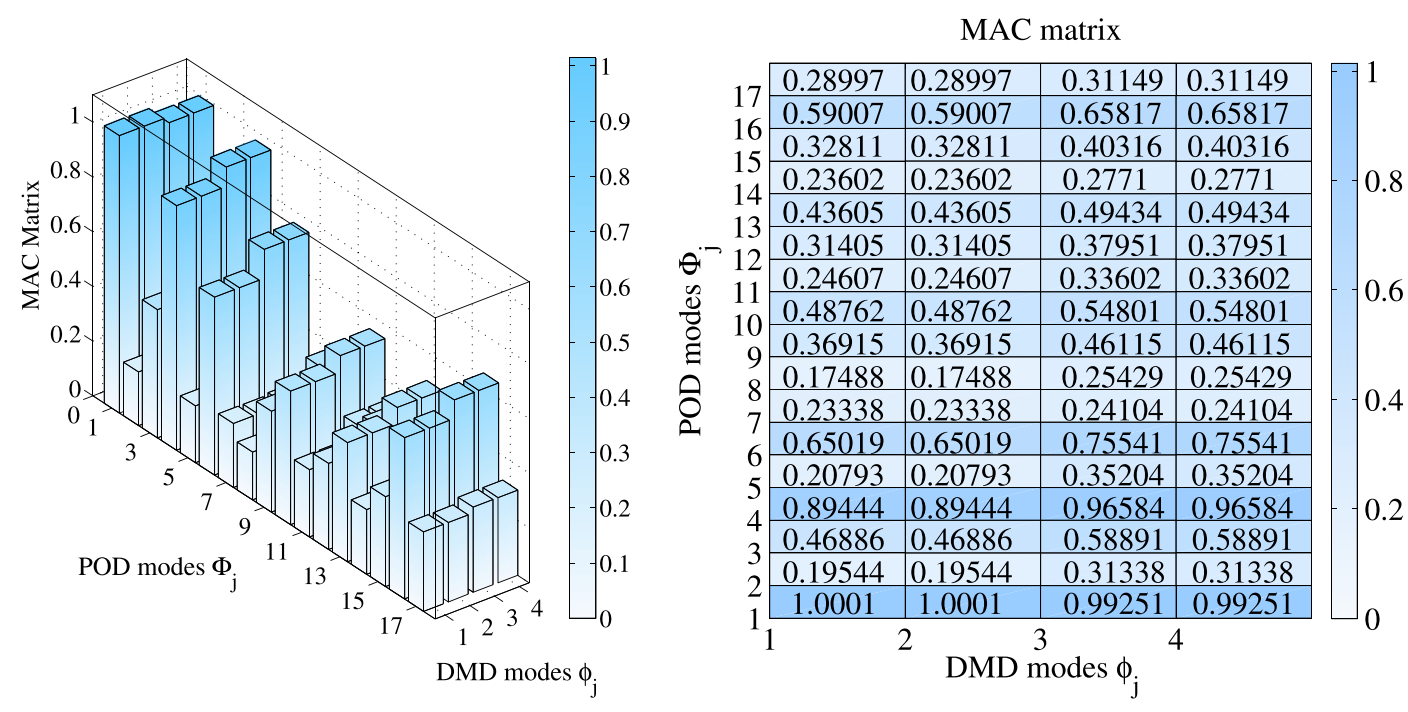

Figure 15. Modal assurance criterion matrix between dynamic mode decomposition and proper orthogonal decomposition modes.

$$
M A C_{i j}\left(\phi_{i}{ }^{D M D}, \Phi_{j}{ }^{P O D}\right)=\frac{\left(\left\|\left(\phi_{j}{ }^{D M D}\right)^{H} \cdot \Phi_{j} P O D\right\|_{F}\right)^{2}}{\left\|\left(\phi_{j}{ }^{D M D}\right)^{H} \cdot{\phi_{j}}^{D M D}\right\|_{F}\left\|\left(\Phi_{j}{ }^{P O D}\right)^{H} \cdot \Phi_{j}{ }^{P O D}\right\|_{F}},
$$

where $\cdot$ represents the Hermitian inner product, $H$ denotes the conjugate transpose and $\|\cdot\|_{F}$ is the Frobenius matrix norm. The computed $M A C_{i j}\left(\phi_{i}{ }^{D M D}, \Phi_{j}{ }^{P O D}\right)$ takes values in the interval $[0,1]$, where 1 indicates identical modes and 0 indicates the orthogonality of the modes. In practice [69], two vectors are considered correlated when the MAC value is greater than 0.9, which corresponds to an angle lower than 18 degrees. The vectors are considered uncorrelated when the MAC value is lower than 0.6, which means that they are separated by an angle greater than 39 degrees.

In the following, we compare the computed DMD modes with the POD modes used as basis functions in the two modal decomposition methods. The MAC values computed between the first $r_{D M D}=4$ modes and the first $r_{P O D}=17$ orthogonal modes are represented in Figure 15.

As expected, the first mode corresponding to the mean flow is well-captured by both methods, with $M A C\left(\phi_{1}{ }^{D M D}, \Phi_{1}{ }^{P O D}\right)=1$. The fourth POD mode $\Phi_{4}$ exhibits a strong similarity with the DMD modes, having an increased MAC value $M A C_{41}=0.89, M A C_{42}=0.89, M A C_{43}=0.96$, $M A C_{44}=0.96$. Analysing the modal assurance matrix, we conclude that only four POD modes are correlated with the DMD modes, namely $\Phi_{1}, \Phi_{4}, \Phi_{6}$ and $\Phi_{16}$, exhibiting a MAC number greater than 0.59. The other POD modes differ from the DMD modes, as with all, they ensure the caption of $99.99 \%$ of the snapshots energy in the POD modal decomposition.

Unlike POD, it is evident that the first four optimal DMD modes are sufficient to describe the flow field, as indicated the higher MAC values between the second, third and fourth DMD modes and the first POD mode: $M A C_{21}=1, M A C_{31}=0.99, M A C_{41}=0.99$. Hence, the conclusion that for the problem investigated here, the DMD modal decomposition is more efficient than POD decomposition, because the DMD modal decomposition is achieved with a smaller number of terms.

\section{ANALYSIS OF OPTIMIZED DYNAMIC MODE DECOMPOSITION-REDUCED ORDER MODEL (ROM) AND PROPER ORTHOGONAL DECOMPOSITION-ROM MODELS}

The two-stage finite-element Numerov-Galerkin method for integrating the nonlinear SWE on a $\beta$-plane limited-area domain proposed by Navon [35] was employed in order to obtain the numerical solution of the SWE model (3)-(5). In Figure 16, the initial velocity fields are presented. The solutions of geopotential height field and $(u, v)$ field at $T=24 \mathrm{~h}$ are illustrated in Figure 17. 

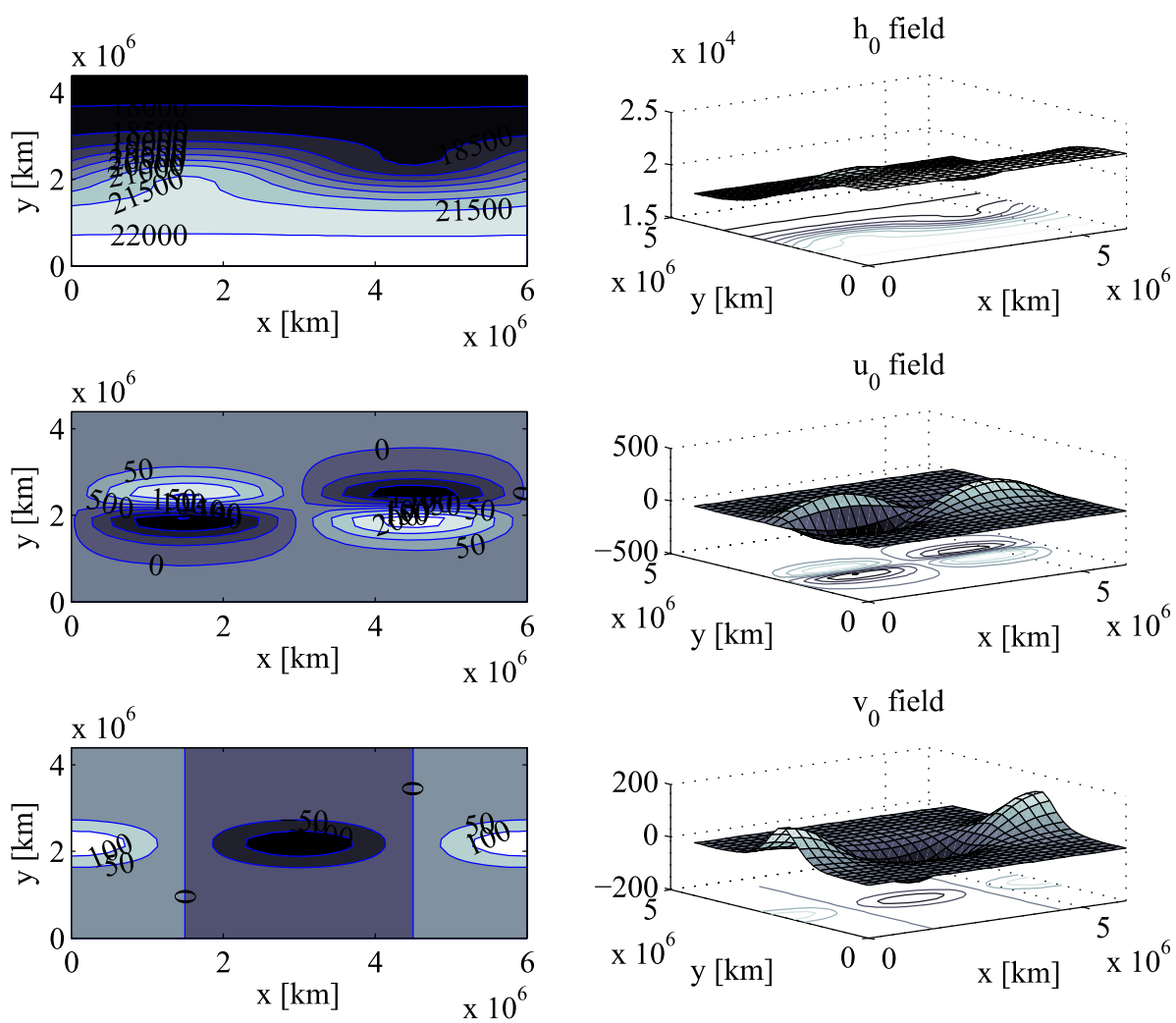

Figure 16. Initial velocity fields: Geopotential height field for the Grammeltvedt initial condition $h_{0}$, streamwise and spanwise velocity fields $\left(u_{0}, v_{0}\right)$ calculated from the geopotential field by using the geostrophic approximation.
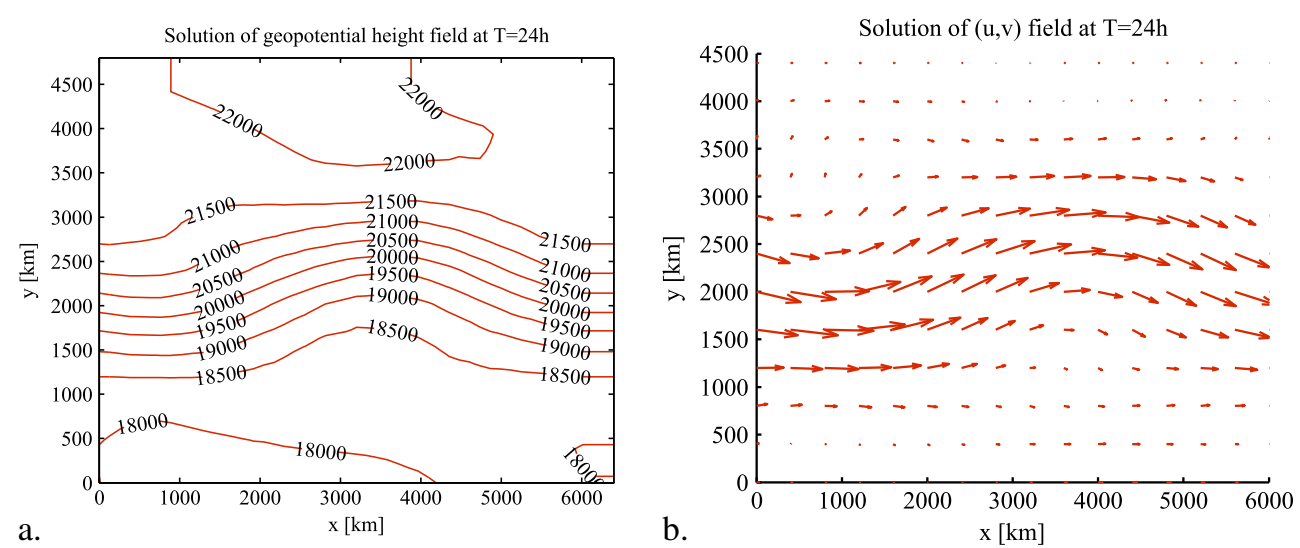

Figure 17. (a) Solution of geopotential height field at $T=24 h$ and (b) solution of ( $u, v$ ) field at $T=24 h$.

The DMD and POD computed geopotential height field and $(u, v)$ field at $T=24 h$ are depicted in Figures 18 and 19, where a number of $r_{D M D}=4$ modes and $r_{P O D}=17$ modes were kept for DMD and POD decompositions, respectively, using snapshots captured at $\Delta t=1200 \mathrm{~s}$.

These reconstructions, when plotted on the same length and time scales as the simulations of the full system, exhibit strikingly similar features, both quantitatively and qualitatively. The validity of the improved DMD approach and POD decomposition has been checked by comparing our results with those obtained by Stefanescu and Navon [13], when an alternating direction fully implicit finite-difference scheme was used for discretization of 2-D SWE on a $\beta$-plane. The flow 

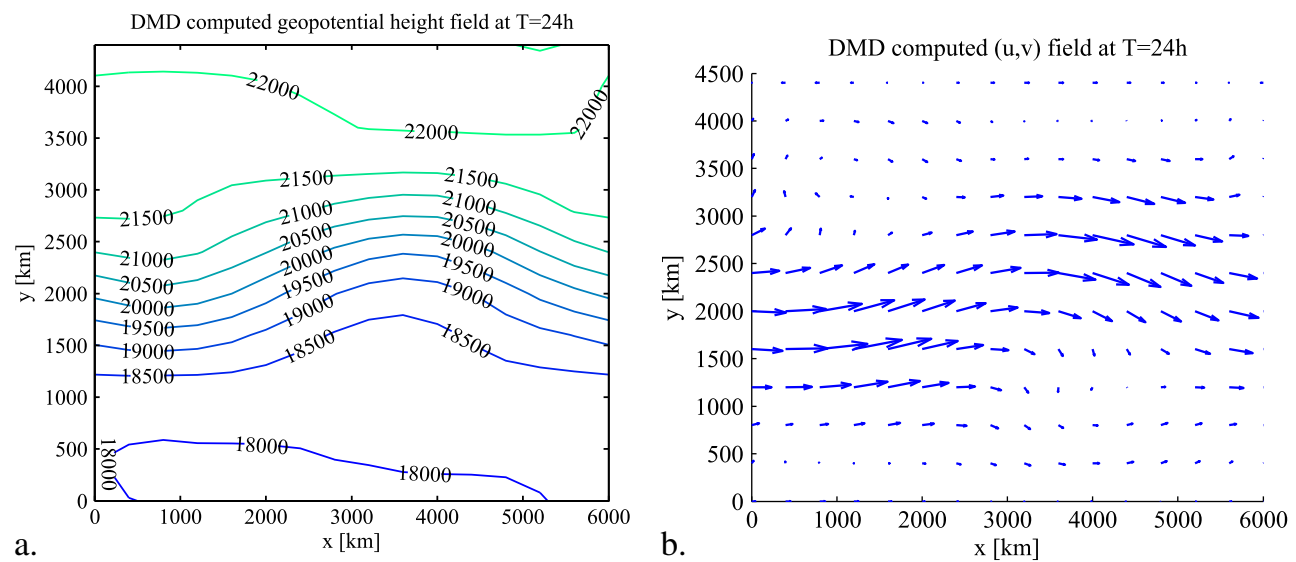

Figure 18. (a) Dynamic mode decomposition (DMD) computed geopotential height field at $T=24 \mathrm{~h}$ and (b) DMD computed $(u, v)$ field at $T=24 h$.
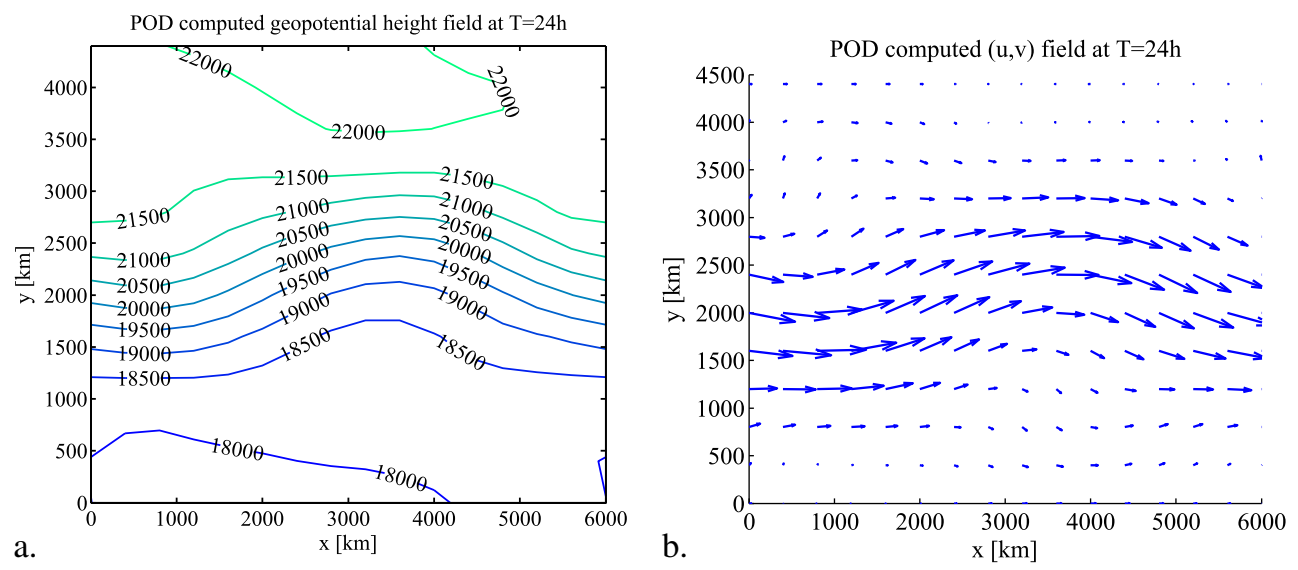

Figure 19. (a) Proper orthogonal decomposition (POD) computed geopotential height field at $T=24 \mathrm{~h}$ and (b) POD computed $(u, v)$ field at $T=24 h$.

reconstructions presented in Figures 18 and 19 are very close to those computed in [13] (see p. 103, Figure 2(a) indicates the results used in comparison).

The similarity between these characteristics of the geopotential height field and those obtained in the previous investigation validates the method presented here and certifies that the improved DMD can be applied successfully to 2-D flows.

We focus in this section on employing tools of DMD, POD and Galerkin projection to provide a consistent way for producing ROM from data.

By collecting snapshots of the velocity and geopotential height field and applying the improved DMD method, an ROM (denoted in the following as DMD-ROM) of the flow is constructed from the DMD basis by writing

$$
w(x, y, t) \approx w^{D M D}(x, y, t)=W_{b}(x, y)+\sum_{j=1}^{r_{D M D}} a_{j}(t) \lambda_{j} \phi_{j}(x, y),
$$

where $W_{b}$ is the centering trajectory, $r_{D M D}$ is the number of DMD basis functions and $\lambda_{j}, \phi_{j}(x, y)$ represent the Ritz eigenvalues of the Koopman operator and the DMD basis functions, respectively. We now replace the velocity $w$ with $w^{D M D}$ in the SWE model (3)-(5) associated with the initial conditions (8), (9), (10), compactly written 


$$
\left\{\begin{array}{l}
\frac{\partial w}{\partial t}(x, y, t)=f(t, w(x, y, t)) \\
w\left(x, y, t_{0}\right)=w_{0}(x, y)
\end{array}\right.
$$

and then project the resulting equations onto the subspace $X^{D M D}=\operatorname{span}\left\{\phi_{1}(\cdot), \phi_{2}(\cdot), \ldots\right.$, $\left.\phi_{r_{D M D}}(\cdot)\right\}$ spanned by the DMD basis to compute the following inner products:

$$
\begin{gathered}
\left\langle\phi_{i}(\cdot), \sum_{j=1}^{r_{D M D}} \lambda_{j} \phi_{j}(\cdot) \dot{a}_{j}(t)\right\rangle=\left\langle\phi_{i}(\cdot), f\left(t, \sum_{j=1}^{r_{D M D}} \lambda_{j} \phi_{j}(\cdot) a_{j}(t)\right)\right\rangle, \\
\left\langle\phi_{i}(\cdot), \sum_{j=1}^{r_{D M D}} \lambda_{j} \phi_{j}(\cdot) \dot{a}_{j}\left(t_{0}\right)\right\rangle=\left\langle\phi_{i}(\cdot), w_{0}\right\rangle, \text { for } i=1 . . r_{D M D},
\end{gathered}
$$

where $\langle f, g\rangle=\int_{\Omega} f g d \Omega$.

The Galerkin projection gives the DMD-ROM, that is, a dynamical system for temporal coefficients $\left\{a_{j}(t)\right\}_{j=1, \ldots, r_{D M D}}$ :

$$
\dot{a}_{i}(t)=\left\langle\phi_{i}(\cdot), f\left(t, \sum_{j=1}^{r_{D M D}} \lambda_{j} \phi_{j}(\cdot) a_{j}(t)\right)\right\rangle,
$$

with the initial condition

$$
a_{i}\left(t_{0}\right)=\left\langle\phi_{i}(\cdot), w_{0}\right\rangle, \text { for } i=1, \ldots, r_{D M D} .
$$

To derive the ROM from the POD basis, denoted in the following as POD-ROM model, we construct the flow by writing

$$
w(x, y, t) \approx w^{P O D}(x, y, t)=W_{b}(x, y)+\sum_{j=1}^{r_{P O D}} b_{j}(t) \Phi_{j}(x, y),
$$

where $W_{b}$ is the centering trajectory, $r_{P O D}$ is the number of POD basis functions and $\Phi_{j}(x, y)$ represents the POD basis functions. We seek for the coefficients $b_{j}$ projecting the SWE equations (51) onto the subspace $X^{P O D}=\operatorname{span}\left\{\Phi_{1}(\cdot), \Phi_{2}(\cdot), \ldots, \Phi_{r_{P O D}}(\cdot)\right\}$ spanned by the POD basis:

$$
\begin{gathered}
\left\langle\Phi_{i}(\cdot), \sum_{j=1}^{r_{P O D}} \Phi_{j}(\cdot) \dot{b}_{j}(t)\right\rangle=\left\langle\Phi_{i}(\cdot), f\left(t, \sum_{j=1}^{r_{P O D}} \Phi_{j}(\cdot) b_{j}(t)\right)\right\rangle, \\
\left\langle\Phi_{i}(\cdot), \sum_{j=1}^{r_{P O D}} \Phi_{j}(\cdot) \dot{b}_{j}\left(t_{0}\right)\right\rangle=\left\langle\Phi_{i}(\cdot), w_{0}\right\rangle .
\end{gathered}
$$

The POD-ROM given by the Galerkin projection reduces to the solution of the following system of ODEs, for the temporal coefficients $\left\{b_{j}(t)\right\}_{j=1, \ldots, r_{P O D}}$ :

$$
\dot{b}_{i}(t)=\left\langle\Phi_{i}(\cdot), f\left(t, \sum_{j=1}^{r_{P O D}} \Phi_{j}(\cdot) b_{j}(t)\right)\right\rangle,
$$

with the initial condition

$$
b_{i}\left(t_{0}\right)=\left\langle\Phi_{i}(\cdot), w_{0}\right\rangle, \text { for } i=1, \ldots, r_{P O D} .
$$


The resulting autonomous systems have linear and quadratic terms parameterized by $c_{i m}, c_{i m n}$, $d_{i m}, d_{i m n}$, respectively:

$$
\begin{gathered}
\dot{a}_{i}(t)=\sum_{m=1}^{r_{D M D}} \sum_{n=1}^{r_{D M D}} c_{i m n} a_{m}(t) a_{n}(t)+\sum_{m=1}^{r_{D M D}} c_{i m} a_{m}(t), \quad i=1, \ldots, r_{D M D}, \\
\dot{b}_{i}(t)=\sum_{m=1}^{r_{P O D}} \sum_{n=1}^{r_{P O D}} d_{i m n} b_{m}(t) b_{n}(t)+\sum_{m=1}^{r_{P O D}} d_{i m} b_{m}(t), \quad i=1, \ldots, r_{P O D} .
\end{gathered}
$$

In the following, we emphasize the performances of the reduced order DMD-ROM model and POD-ROM model for 2-D flows in comparison with the numerical solution of the full SWE model. To judge the quality of the ROMs developed here, an error estimate is provided. We define the relative error as the $L_{2}$-norm of the difference between the variables of the full SWE model and approximate solutions over the exact one, that is,

$$
\begin{gathered}
\operatorname{error}_{D M D}=\frac{\left\|w(x, y)-w^{D M D-R O M}(x, y)\right\|_{2}}{\|w(x, y)\|_{2}}, \\
\operatorname{error}_{P O D}=\frac{\left\|w(x, y)-w^{P O D-R O M}(x, y)\right\|_{2}}{\|w(x, y)\|_{2}} .
\end{gathered}
$$

The results are presented in Table I. The maximum error of numerical POD-ROM solutions is less than the error of numerical DMD-ROM solutions, but the benefit of employing the improved DMD

Table I. The average relative errors of reduced order models.

\begin{tabular}{ll}
\hline DMD-ROM & POD-ROM \\
\hline error $_{D M D}{ }^{h}=0.0119$ & error $P O D^{h}=0.0042$ \\
error $_{D M D} u=0.1770$ & error $_{P O D}^{u}=0.0929$ \\
error $_{D M D}=0.1534$ & error $P O D^{v}=0.0456$ \\
\hline
\end{tabular}

DMD, dynamic mode decomposition; POD, proper orthogonal decomposition, ROM, reduced order model.

Table II. Energy conserving test.

\begin{tabular}{lr}
\hline Absolute error of DMD reduced order model & Absolute error of POD reduced order model \\
\hline$\left|E-E^{D M D}\right|=0.1956 \times 10^{-5}$ & $\left|E-E^{P O D}\right|=0.7436 \times 10^{-6}$ \\
\hline
\end{tabular}

DMD, dynamic mode decomposition; POD, proper orthogonal decomposition.
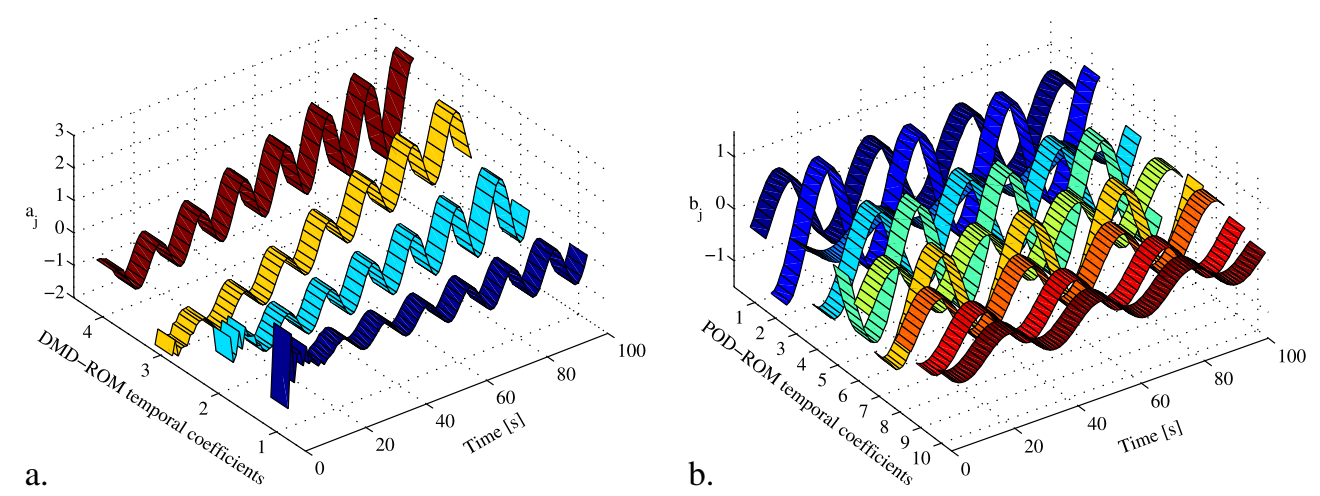

Figure 20. (a) Dynamic mode decomposition-reduced order model (ROM) computed temporal coefficients; (b) proper orthogonal decomposition-ROM computed temporal coefficients. 

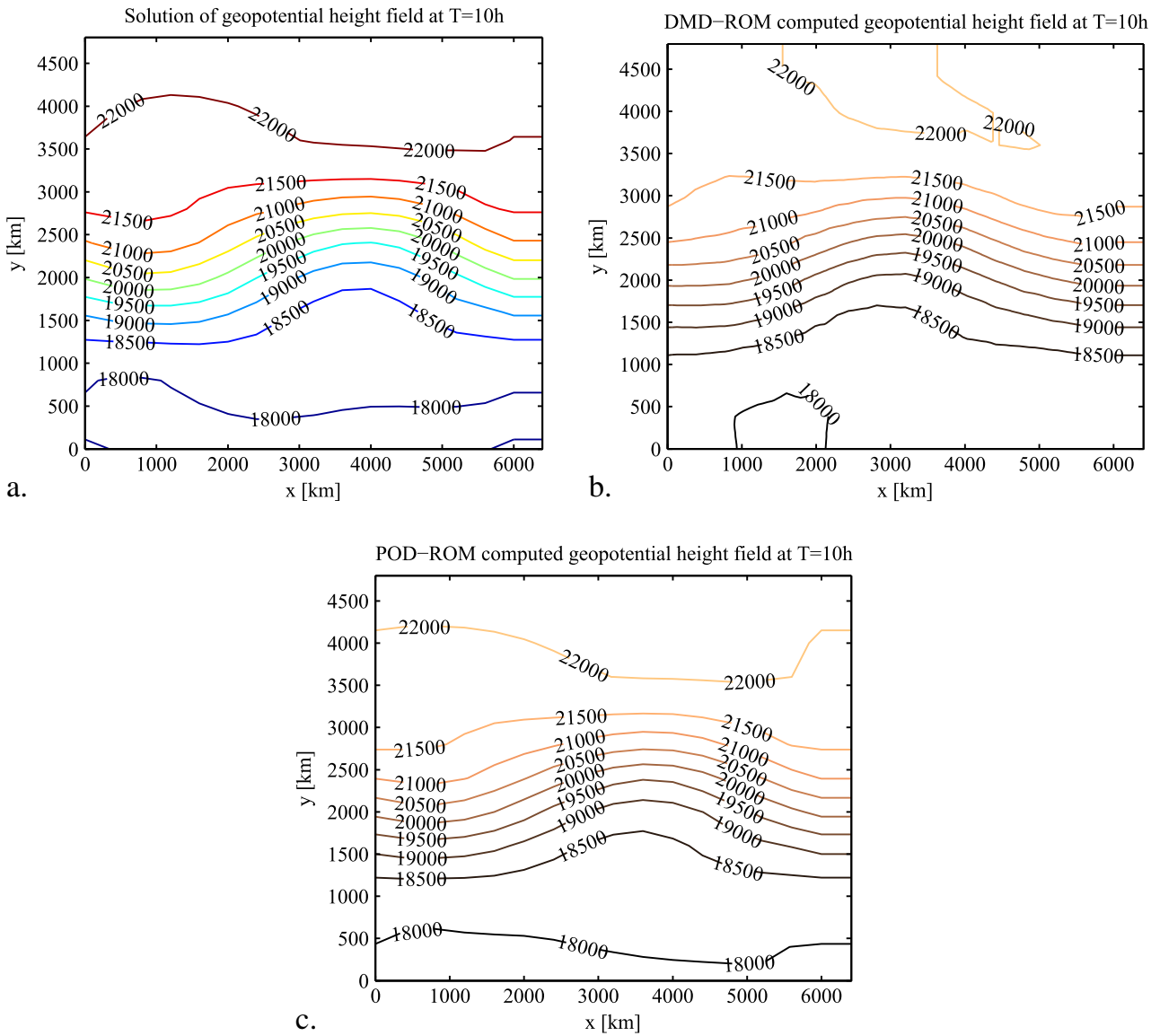

Figure 21. Comparison of the geopotential height field between full model and reduced order models (ROM): (a) Solution of geopotential height field computed at time $T=10 \mathrm{~h}$; (b) dynamic mode decomposition-ROM solution of geopotential height field computed at time $T=10 h$; and (c) proper orthogonal decomposition-ROM solution of geopotential height field computed at time $T=10 \mathrm{~h}$.
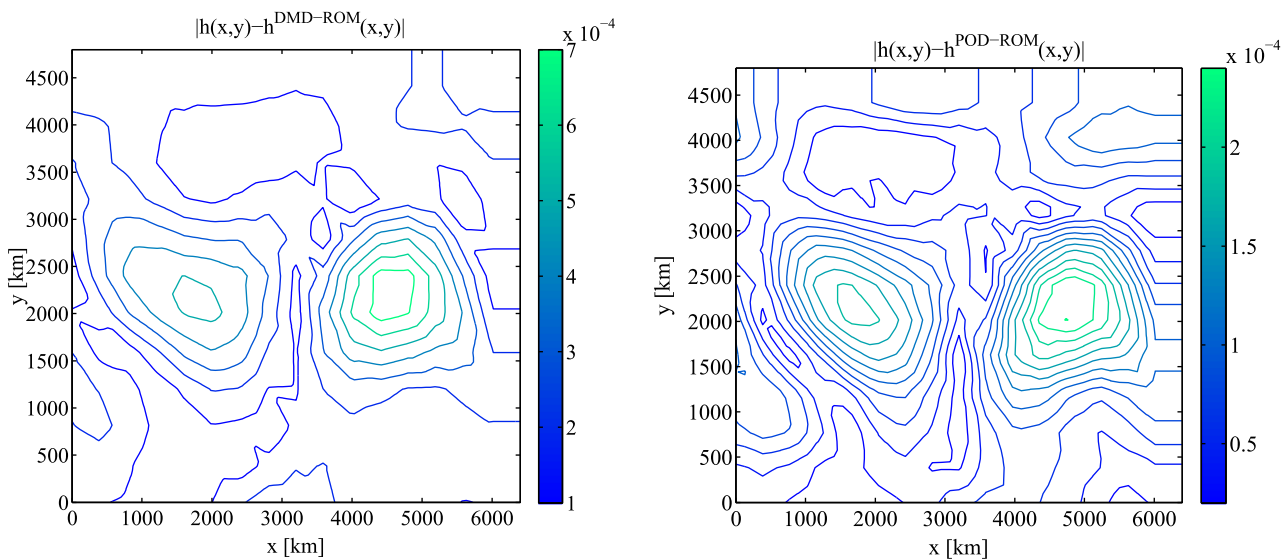

Figure 22. Local errors between dynamic mode decomposition-reduced order model (ROM), proper orthogonal decomposition-ROM shallow water equations (SWE) solutions and the full SWE solution at time $T=10 h$. 
prevails in the case of SWE model reduction. Although the POD-ROM model provides higher precision, the DMD-ROM model is less expensive with respect to the numerical implementation costs, that is, numerical results are obtained for a considerably smaller number of expansion terms to derive the ROM.

The flow energy conservation is used as an additional metric to evaluate the quality of the two reduced order models. Table II presents the absolute errors with respect to flow energy obtained from the full model. The results indicate that both ROMs will preserve the flow total energy.

Figure 20 illustrates the temporal coefficients of the two ROMs. In Figure 20a, the coefficients of the DMD-ROM model, corresponding to the first four dominant Koopman modes are visualized. Figure 20b plots the coefficients corresponding to the first ten POD modes of the POD-ROM model.

A comparison of the full solution of the geopotential height field and ROMs solutions is provided in Figure 21. The geopotential height field computed at time level $T=10 \mathrm{~h}$ by the two ROMs exhibits an overall good agreement with that from the full model.

The local error between the full SWE solution and DMD-ROM and POD-ROM solutions, respectively, at time $T=10 \mathrm{~h}$ is presented in Figure 22 .

The correlation coefficients defined below are used as additional metrics to validate the quality of the two ROMs:

$$
\begin{gathered}
C_{i}{ }^{D M D}=\frac{\left(\left\|w_{i}(x, y) \cdot w_{i}{ }^{D M D-R O M}(x, y)\right\|_{F}\right)^{2}}{\left\|\left(w_{i}(x, y)\right)^{H} \cdot w_{i}(x, y)\right\|_{F}\left\|\left(w_{i}{ }^{D M D-R O M}(x, y)\right)^{H} \cdot w_{i}{ }^{D M D-R O M}(x, y)\right\|_{F}}, \\
i=0, \ldots, N-1, \\
C_{i}{ }^{P O D}=\frac{\left(\left\|w_{i}(x, y) \cdot w_{i}{ }^{P O D-R O M}(x, y)\right\|_{F}\right)^{2}}{\left\|\left(w_{i}(x, y)\right)^{H} \cdot w_{i}(x, y)\right\|_{F}\left\|\left(w_{i}{ }^{P O D-R O M}(x, y)\right)^{H} \cdot w_{i}{ }^{P O D-R O M}(x, y)\right\|_{F}}, \\
i=0, \ldots, N-1,
\end{gathered}
$$

where $w_{i}(x, y)$ means the solution of the full SWE model at time $i, w_{i}{ }^{D M D-R O M}(x, y)$, $w_{i}^{P O D-R O M}(x, y, t)$ represent the computed solutions at time $i$ by means of the ROMs, respectively, (.) represents the Hermitian inner product and $H$ denotes the conjugate transpose. A comparison of the correlation coefficients between the full model and ROMs is provided in Figure 23. The values of the correlation coefficients are greater than $99 \%, 97 \%$, respectively, and confirm the validity of the two ROMs.
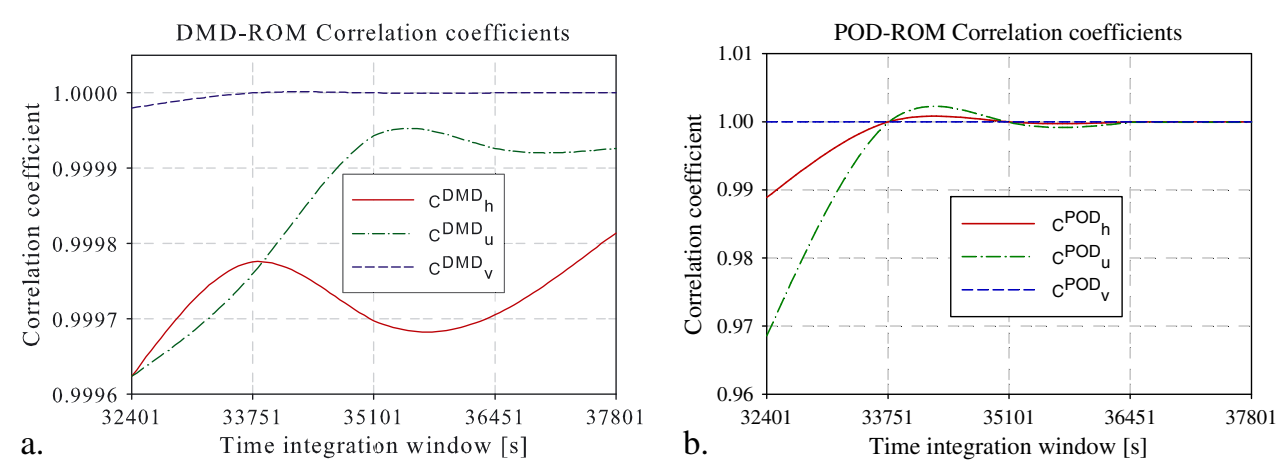

Figure 23. Correlation coefficients for the shallow water equations (SWE) variables: (a) dynamic mode decomposition-reduced order model (ROM) versus full SWE model; (b) proper orthogonal decompositionROM model versus full SWE model. 


\section{SUMMARY AND CONCLUSIONS}

We have proposed a framework for DMD of 2-D flows, when numerical or experimental data snapshots are captured with large time steps. Such problems originate for instance from meteorology, when a large time step acts like a filter in obtaining the significant Koopman modes, therefore the classic DMD method is not effective. This study was motivated by the need to further clarify the connection between Koopman modes and POD dynamic modes. We have applied DMD and POD to derive ROMs of the SWE.

Based on the DMD method introduced in [50], we proposed an improved DMD algorithm for selecting the dominant Koopman modes of the flow field. Unlike the classic algorithm, we arrange the Koopman modes in descending order of the energy of the DMD modes weighted by the inverse of the Strouhal number. Key innovations for the DMD-based ROM introduced in this paper are the use of the Moore-Penrose pseudoinverse in the DMD computation that produced an accurate result and a novel selection method for the DMD modes and associated amplitudes and Ritz values. We eliminate the modes that contribute weakly to the data sequence based on the conservation of quadratic integral invariants by the reduced order flow.

In order to assess the performances of the proposed method, we have considered two numerical experiments, and we applied the improved DMD algorithm for different snapshots obtained by sampling down the original solutions of the full SWE model with different time steps. We compared the novel approach with the classic one in both cases. The improved DMD algorithm introduced in this paper proved to be more efficient for model reduction than the classic DMD method. Solution of the constrained optimization problem (40) leads to the number of $r_{D M D}=23, r_{D M D}=21$ Koopman modes in the case of the classic DMD algorithm, respectively, while a number of $r_{D M D}=$ $13, r_{D M D}=4$ Koopman modes, respectively, are kept in the case of the improved DMD algorithm for flow modal decomposition in the two considered experiments.

We emphasized the excellent behaviour of the improved DMD method compared with PODbased model results. Following the classic energetic criterion (45), the POD decomposition leads to a number of $r_{P O D}=17$ selected modes. We perform a quantitative comparison of the spatial modes computed from the two decompositions discussed here using the MAC as a measure of the degree of linearity between Koopman and POD modes. This evaluation indicates that the DMD modal decomposition is more efficient than POD decomposition because the DMD modal decomposition is achieved with a smaller number of modes.

Additionally, we presented a rigorous error analysis for the ROM models obtained by POD and the improved DMD, and we compared the relative computational efficiency of the aforementioned ROM methods.

We found a very close agreement between the flow reconstruction computed with the ROM models and the solution provided by the high fidelity SWE model. But the benefit of employing the improved DMD method prevails in the case of modal decomposition of 2-D flows described by SWE. The similarity between the correlation coefficients between the full solution and the reduced order solutions certifies that the improved DMD method can be applied successfully in parallel with the POD decomposition to obtain ROMs of potential relevance.

Finally, let us summarize the main features of the presented methods and potential applications of the novel numerical method introduced in this paper:

(i) Improved DMD method introduced in the present research exhibits more efficiency in reconstruction of flows described by SWE model. For $\Delta t=1200 \mathrm{~s}, r_{D M D}=4$ Koopman modes are selected for flow reconstruction, while $r_{D M D}=21$ Koopman modes are retained in the case of the classic DMD algorithm and $r_{P O D}=17$ modes are kept for flow reconstruction in POD method.

(ii) By employing the DMD, the most energetic Koopman modes are associated to the higher amplitudes selected for flow decomposition. Instead, the eigenvalues capturing most of the snapshots energy indicate the corresponding basis functions in POD decomposition.

(iii) DMD is useful when the main interest is to capture the dominant frequency of the phenomenon. POD is useful when the main interest is to find coherent structures in the POD 
modes, which are energetically ranked. Further techniques for system identification or flow optimization can be addressed based on both DMD method and POD method.

The question whether the proposed DMD methodology is a viable alternative to the linear stability analysis available in the community for hydrodynamic stability investigation is a subject that will be addressed carefully in our future work. There are a number of interesting directions that arise from this work. First, it will be a natural extension to apply the proposed algorithm to high-dimensional systems in fluid dynamics and to oceanographic/atmospheric measurements. The methodology presented here offers the main advantage of deriving an ROM capable to provide a variety of information describing the behavior of 2-D flows. A future extension of this research will address an efficient numerical approach for modal decomposition of swirling flows, where the full mathematical model implies more sophisticated relations at domain boundaries that must be satisfied by the ROM also.

Projection-based methods presented in this paper lead to ROMs with dramatically reduced numbers of equations and unknowns. However, for parametrically varying problems or for modelling problems with strong nonlinearities, the cost of evaluating the reduced order models still depends on the size of the full order model and is therefore still expensive. The discrete empirical interpolation method (DEIM) described in detail in [70] further approximates the nonlinearity in the projection based reduced order strategies. The application of a DEIM-ROM strategy for finite element (FEM) models combined with the methods proposed in this paper represents a subject that we will further address in our studies. The resulting DEIM-DMD-ROM and DEIM-POD-ROM will be evaluated efficiently at a cost that is independent of the size of the original problem.

\section{ACKNOWLEDGEMENTS}

The first author acknowledges the partial support of strategic grant POSDRU/159/1.5/S/137070 (2014) of the Ministry of National Education, Romania, co-financed by the European Social Fund-Investing in People, within the Sectorial Operational Program Human Resources Development 2007-2013.

Prof. I.M. Navon acknowledges the support of NSF grant ATM-0931198. The authors would like to thank the anonymous reviewers who greatly contributed in improving the presentation of the present paper.

\section{REFERENCES}

1. Holmes PJ, Lumley JL, Berkooz G, Rowley CW. Turbulence, Coherent Structures, Dynamical Systems and Symmetry (2nd edition). Cambridge University Press: Cambridge, UK, 2011.

2. Ouellette NT. On the dynamical role of coherent structures in turbulence. Comptes Rendus Physique 2012; 13: 866-877.

3. Liao Y, Ouellette NT. Spatial structure of spectral transport in two-dimensional flow. Journal of Fluid Mechanics 2013; 725:281-298.

4. Luchtenburg DM, Rowley CW. Model reduction using snapshot-based realizations. American Physical Society, 64th Annual Meeting of the APS Division of Fluid Dynamics 2011; 56(18):Abstract H19.00004.

5. Liberge E, Hamdouni A. Reduced order modelling method via proper orthogonal decomposition (POD) for flow around an oscillating cylinder. Journal of Fluids and Structures 2010; 26:292-311.

6. Wang Z, Akhtar I, Borggaard J, Iliescu T. Proper orthogonal decomposition closure models for turbulent flows: a numerical comparison. Computer Methods in Applied Mechanics and Engineering 2012; 237-240:10-26.

7. Abramov RV, Majda AJ. Low-frequency climate response of quasigeostrophic wind-driven ocean circulation. Journal of Physical Oceanography 2012; 42:243-260.

8. Osth J, Noack BR, Krajnovic S, Barros D, Boree J. On the need for a nonlinear subscale turbulence term in POD models as exemplified for a high-Reynolds-number flow over an Ahmed body. Journal of Fluid Mechanics 2014; 747:518-544.

9. Mariani R, Dessi D. Analysis of the global bending modes of a floating structure using the proper orthogonal decomposition. Journal of Fluids and Structures 2012; 28:115-134.

10. Buljak V, Maier G. Proper orthogonal decomposition and radial basis functions in material characterization based on instrumented indentation. Engineering Structures 2011; 33:492-501.

11. Du J, Fang F, Pain CC, Navon IM, Zhu J, Ham D. POD reduced order unstructured mesh modelling applied to 2D and 3D fluid flow. Computers and Mathematics with Applications 2013; 65:362-379.

12. Fang F, Pain CC, Navon IM, Piggott MD, Gorman GJ, Allison P, Goddard AJH. Reduced order modelling of an adaptive mesh ocean model. International Journal for Numerical Methods in Fluids 2009; 59(8):827-851.

13. Stefanescu R, Navon IM. POD/DEIM nonlinear model order reduction of an ADI implicit shallow water equations model. Journal of Computational Physics 2013; 237:95-114. 
14. Winton C, Pettway J, Kelley CT, Howington S, Eslinger OJ. Application of proper orthogonal decomposition (POD) to inverse problems in saturated groundwater flow. Advances in Water Resources 2011; 34:1519-1526.

15. Chen X, Navon IM, Fang F. A dual-weighted trust-region adaptive POD 4D-VAR applied to a finite-element shallowwater equations model. International Journal for Numerical Methods in Fluids 2011; 65:250-541.

16. Chen X, Akella S, Navon IM. A dual-weighted trust-region adaptive POD 4-D VAR applied to a finite-volume shallow water equations model on the sphere. International Journal for Numerical Methods in Fluids 2012; 68: $377-402$.

17. Cao Y, Zhu J, Luo Z, Navon IM. Reduced order modeling of the upper tropical Pacific Ocean model using proper orthogonal decomposition. Computers and Mathematics with Applications 2006; 52(8-9):1373-1386.

18. Cao Y, Zhu J, Navon IM, Luo Z. A reduced order approach to four-dimensional variational data assimilation using proper orthogonal decomposition. International Journal for Numerical Methods in Fluids 2007; 53(10):1571-1583.

19. Ilak M, Schlatter P, Bagheri S, Henningson DS. Bifurcation and stability analysis of a jet in cross-flow: onset of global instability at a low velocity ratio. Journal of Fluid Mechanics 2012; 696:94-121.

20. Moore C. Principal component analysis in linear systems: controllability, observability, and model reduction. IEEE Transactions on Automatic Control 1981; 26:17-32.

21. Rowley CW. Model reduction for fluids, using balanced proper orthogonal decomposition. International Journal of Bifurcation and Chaos 2005; 15(3):997-1013.

22. Bagheri S. Koopman-mode decomposition of the cylinder wake. Journal of Fluid Mechanics 2013; 726:596-623.

23. Mezic I. Analysis of fluid flows via spectral properties of the Koopman operator. Annual Review of Fluid Mechanics 2013; 45(1):357-378.

24. Rowley CW, Mezic I, Bagheri S, Schlatter P. Henningson DS. Spectral analysis of nonlinear flows. Journal of Fluid Mechanics 2009; 641:115-127.

25. Koopman B. Hamiltonian systems and transformations in Hilbert space. Proceedings of the National Academy of Sciences $1931 ; \mathbf{1 7}: 315-318$.

26. Semeraro O, Bellani G, Lundell F. Analysis of time-resolved PIV measurements of a confined turbulent jet using POD and Koopman modes. Experiments in Fluids 2012; 53:1203-1220.

27. Soucasse L, Riviere P, Soufiani A, Xin S, Quere PL. Transitional regimes of natural convection in a differentially heated cubical cavity under the effects of wall and molecular gas radiation. Physics of Fluids 2014; 26:024105.

28. Frederich O, Luchtenburg DM. Modal analysis of complex turbulent flow. The 7th International Symposium on Turbulence and Shear Flow Phenomena (TSFP-7): Ottawa, Canada, 2011.

29. Muld TW, Efraimsson G, Henningson DS. Flow structures around a high-speed train extracted using proper orthogonal decomposition and dynamic mode decomposition. Computers and Fluids 2012; 57:87-97.

30. Hossen MJ, Navon IM, Fang F. A penalized four-dimensional variational data assimilation method for reducing forecast error related to adaptive observations. International Journal for Numerical Methods in Fluids 2012; 70: $1207-1220$.

31. Daescu DN, Navon IM. Adaptive observations in the context of 4D-VAR data assimilation. Meteorology and Atmospheric Physics 2004; 85:205-226.

32. San O, Staples A, Wang Z, Iliescu T. Approximate deconvolution large eddy simulation of a barotropic ocean circulation model. Ocean Modelling 2011; 40(2):120-132.

33. Iliescu T, Fischer PF. Large eddy simulation of turbulent channel flows by the rational large eddy simulation model. Physics of Fluids 2003; 15:3036-3047.

34. Vreugdenhil CB. Numerical Methods for Shallow Water Flow. Kluwer Academic Publishers: Dordrecht, 1994.

35. Navon IM. FEUDX: A two-stage, high accuracy, finite-element Fortran program for solving shallow-water equations. Computers and Geosciences 1987; 13(3):255-285.

36. Bryden IG, Couch SJ, Owen A, Melville G. Tidal current resource assessment. Proceedings of the Institution of Mechanical Engineers, Part A: Journal of Power and Energy 2007; 221:125-135.

37. Sportisse B, Djouad R. Reduction of chemical kinetics in air pollution modeling. Journal of Computational Physics 2000; 164:354-376.

38. Koutitus C. Mathematics models in coastal engineering. Pentech Press: London, 1988.

39. Grammeltvedt A. A survey of finite-diference schemes for the primitive equations for a barotropic fluid. Monthly Weather Review 1969; 97(5):384-404.

40. Cullen MJP, Morton KW. Analysis of evolutionary error in finite-element and other methods. Journal of Computational Physics 1980; 34:245-267.

41. Navon IM. Finite-element simulation of the shallow-water equations model on a limited area domain. Applied Mathematical Modeling 1979; 3:337-348.

42. Fang F, Pain CC, Navon IM, Cacuci DG, Chen X. The independent set perturbation method for efficient computation of sensitivities with applications to data assimilation and a finite element shallow water model. Computers and Fluids 2013; 76:33-49.

43. Navon IM, Phua PKH, Ramamurthy M. Vectorization of conjugate-gradient methods for large-scale minimization in meteorology. Journal of Optimization Theory and Applications 1990; 66(1):71-93.

44. Navon IM, DeVilliers R. GUSTAF: a quasi-Newton nonlinear ADI Fortran IV program for solving the shallow-water equations with augmented Lagrangians. Computers and Geosciences 1986; 12:151-173.

45. Navon IM. A Numerov-Galerkin technique applied to a finite-element shallow water equations model with enforced conservation of integral invariants and selective lumping. Journal of Computational Physics 1983; 52:313-339. 
46. Arakawa A, Hsu YJG. Energy conserving and potential-enstrophy dissipating schemes for the shallow water equations. Monthly Weather Review 1990; 118:1960-1969.

47. Arakawa A. Computational design for long-term numerical integration of the equations of fluid motion: twodimensional incompressible flow. Part I. Journal of Computational Physics 1997; 135:103-114.

48. Fiedler M. A note on companion matrices. Linear Algebra and its Applications 2003; 372:325-331.

49. Rowley CW, Mezic I, Bagheri S, Schlatter P, Henningson DS. Reduced-order models for flow control: balanced models and Koopman modes. Seventh IUTAM Symposium on Laminar-Turbulent Transition, IUTAM Bookseries 2010; 18:43-50.

50. Schmid P. Dynamic mode decomposition of numerical and experimental data. Journal of Fluid Mechanics 2010; 656:5-28.

51. Bagheri S. Computational hydrodynamic stability and flow control based on spectral analysis of linear operators. Archives of Computational Methods in Engineering 2012; 19(3):341-379.

52. Golub G, van Loan CF. Matrix Computations (Third Edition). The Johns Hopkins University Press: Baltimore, Maryland, 1996.

53. Schmid PJ, Violato D, Scarano F. Decomposition of Time-Resolved Tomographic PIV. Experiments in Fluids 2012; 52(6): $1567-1579$

54. Duke D, Soria J, Honnery D. An error analysis of the dynamic mode decomposition. Experiments in Fluids 2012; 52:529-542.

55. Bagheri S. Effects of weak noise on oscillating flows: linking quality factor, Floquet modes, and Koopman spectrum. Physics of Fluids 2014; 26:094104-1-094104-17.

56. Noack BR, Afanasiev K, Morzynski M, Tadmor G, Thiele F. A hierarchy of low-dimensional models for the transient and post-transient cylinder wake. Journal of Fluid Mechanics 2003; 497:335-363.

57. Chen KK, Tu JH, Rowley CW. Variants of dynamic mode decomposition: boundary condition, Koopman and Fourier analyses. Journal of Nonlinear Science 2012; 22:887-915.

58. Belson B, Tu JH, Rowley CW. Algorithm 945: Modred - a parallelized model reduction library. ACM Transactions on Mathematical Software 2014; 40(4):Article 30.

59. Holmes P, Lumley JL, Berkooz G. Turbulence, Coherent Structures, Dynamical Systems and Symmetry. Cambridge Univ. Press.: New York, 1996.

60. Sirovich L. Turbulence and the dynamics of coherent structures, parts I-III. Quarterly of Applied Mathematics 1987; 3:561-590.

61. Jovanovic MR, Schmid PJ, Nichols JW. Low-rank and sparse dynamic mode decomposition. Center for Turbulence Research Annual Research Briefs 2012:139-152.

62. Schmid PJ, Meyer KE, Pust O. Dynamic mode decomposition and proper orthogonal decomposition of flow in a liddriven cylindrical cavity. 8th International Symposium on Particle Image Velocimetry - PIV09: Melbourne, Victoria, Australia, 2009.

63. Balajewicz MJ, Dowell EH, Noack BR. Low-dimensional modelling of high-Reynolds-number shear flows incorporating constraints from the Navier-Stokes equation. Journal of Fluid Mechanics 2013; 729:285-308.

64. Noack BR, Morzynski M, Tadmor G. Reduced-Order Modelling for Flow Control. Springer: Wien, 2011.

65. Nyquist H. Certain topics in telegraph transmission theory. Transactions of the American Institute of Electrical Engineers 1928; 47:617-644.

66. Salmon R. A general method for conserving energy and potential enstrophy in shallow-water models. Journal of Atmospheric Science 2007; 64:515-531.

67. Nocedal J, Wright SJ. Numerical Optimization, Second Edition. Springer: New York, 2006.

68. Brown DL, Allemang RJ, Zimmerman RD, Mergeay M. Parameter estimation techniques for modal analysis. $S A E$ Transactions 1979; SAE Paper Number 790221; 88:828-846.

69. Mitchell LD. Increasing the sensitivity of the modal assurance criteria (MAC) to small mode shape changes: The IMAC. Proceedings of the 16th International Modal Analysis Conference, 1998; 64-69.

70. Antil H, Heinkenschloss M, Sorensen D. Reduced Order Methods for Modelng and Computational Reduction, chap. Application of the Discrete Empirical Interpolation Method to Reduced Order Modeling of Nonlinear and Parametric Systems. Springer International Publishing Switzerland, 2014; 101-136. 